\title{
Identification of Aberrantly Expressed Genes during Aging in Rat Nucleus Pulposus Cells
}

\author{
Shi Cheng $\left(\mathbb{D},{ }^{1}\right.$ Xiaochuan $L i,{ }^{2,3}$ Linghan Lin $\mathbb{D},{ }^{4}$ Zhiwei Jia $\mathbb{D},{ }^{5}$ Yachao Zhao $\mathbb{D},{ }^{4}$ \\ Deli Wang $\mathbb{D}^{6,7}$ Dike Ruan $\mathbb{D}^{4},{ }^{4}$ and Yu Zhang $\mathbb{D}^{1}$
}

${ }^{1}$ Department of Orthopedics, Guangdong General Hospital, Guangdong Academy of Medical Science, South China University of Technology, Guangzhou 510080, China

${ }^{2}$ Department of Orthopedic Surgery, Gaozhou People's Hospital, Guangdong 525200, China

${ }^{3}$ Post-Doctoral Innovation Practice Base of Gaozhou People's Hospital, Guangdong 525200, China

${ }^{4}$ Department of Orthopaedics, The Sixth Medical Centre of PLA General Hospital, 100048 Beijing, China

${ }^{5}$ Department of Orthopaedics, The 306th Hospital of People's Liberation Army, Beijing, China

${ }^{6}$ Department of Bone \& Joint Surgery, Peking University Shenzhen Hospital, Shenzhen, 518036 Guangdong, China

${ }^{7}$ National \& Local Joint Engineering Research Center of Orthopaedic Biomaterials, Peking University Shenzhen Hospital, Shenzhen, 518036 Guangdong, China

Correspondence should be addressed to Deli Wang; wangdelinavy@163.com, Dike Ruan; ruandikengh@163.com, and Yu Zhang; luck_2001@126.com

Received 2 March 2019; Revised 6 May 2019; Accepted 30 May 2019; Published 10 July 2019

Guest Editor: Zhen Li

Copyright (c) 2019 Shi Cheng et al. This is an open access article distributed under the Creative Commons Attribution License, which permits unrestricted use, distribution, and reproduction in any medium, provided the original work is properly cited.

Nucleus pulposus cells (NPCs) play a vital role in maintaining the homeostasis of the intervertebral disc (IVD). Previous studies have discovered that NPCs exhibited malfunction due to cellular senescence during disc aging and degeneration; this might be one of the key factors of IVD degeneration. Thus, we conducted this study in order to investigate the altered biofunction and the underlying genes and pathways of senescent NPCs. We isolated and identified NPCs from the tail discs of young ( 2 months) and old (24 months) SD rats and confirmed the senescent phenotype through SA- $\beta$-gal staining. CCK- 8 assay, transwell assay, and cell scratch assay were adopted to detect the proliferous and migratory ability of two groups. Then, a rat Gene Chip Clariom $^{\mathrm{TM}} \mathrm{S}$ array was used to detect differentially expressed genes (DEGs). After rigorous bioinformatics analysis of the raw data, totally, 1038 differentially expressed genes with a fold change $>1.5$ were identified out of 23189 probes. Among them, 617 were upregulated and 421 were downregulated. Furthermore, Gene Ontology (GO) and Kyoto Encyclopedia of Genes and Genomes (KEGG) pathway analysis were conducted and revealed numerous number of enriched GO terms and signaling pathways associated with senescence of NPCs. A protein-protein interaction (PPI) network of the DEGs was constructed using the Search Tool for the Retrieval of Interacting Genes (STRING) database and Cytoscape software. Module analysis was conducted for the PPI network using the MCODE plugin in Cytoscape. Hub genes were identified by the CytoHubba plugin in Cytoscape. Derived 5 hub genes and most significantly up- or downregulated genes were further verified by real-time PCR. The present study investigated underlying mechanisms in the senescence of NPCs on a genomewide scale. The illumination of molecular mechanisms of NPCs senescence may assist the development of novel biological methods to treat degenerative disc diseases. 


\section{Introduction}

Low back pain (LBP) is a major age-related disease, not only contributing to patients' suffering and disability but also causing large financial burden to society [1]. Intervertebral disc degeneration (IVDD) has been confirmed to be one of the most fundamental pathological changes of LBP [2]. Due to a largely unknown mechanism of IVDD, effective therapy methods still need investigation.

Traditional therapy strategies including surgery and conservative therapy are aimed at alleviating symptoms instead of regenerating the degenerated disc. Thus, biological approaches which mainly focus on restoring the structure and function of the IVD are considered more promising $[3,4]$. The structure of the intervertebral disc could be divided into three different regions: nucleus pulposus (NP), annulus fibrosus (AF), and cartilaginous endplate (CEP) [5]. NP is a kind of gelatinous tissue containing extracellular matrix (ECM) comprising highly hydrated proteoglycan, collagen fibers, and aggrecan [6]. It plays a vital role in maintaining the physiological function of IVD because NP could absorb stress when the IVD is confronting diverse mechanical impact [7]. Nucleus pulposus cells (NPCs) are the organ-specific cells residing in the nucleus pulposus [8]. NPCs are responsible for the metabolism homeostasis of the ECM by producing collagen I, collagen II, and proteoglycan, which are the main components of the gelatinous structures of NP [9]. During aging and degeneration of IVD, the normal function of NPCs was disrupted, thus resulting in aberrant metabolism of ECM, which could accelerate the process of IVDD $[10,11]$. Cytotherapy by reactivate degenerated NPCs has been proposed to be an ideal biological therapy method to treat IVDD [11]. However, the specific mechanism of NPC degeneration is still unknown, which hindered the development of cytotherapy.

Cell senescence is defined as a cellular program that leads to a stable growth arrest along with distinct phenotypic alterations and presentation of senescence-associated secretory phenotype (SASP) $[12,13]$. Senescence of disc cells has been widely accepted as one of the major factors of IVD degeneration and aging [14, 15]. The number of viable cell in NP was decreased, and the cellular function of NPCs is being impaired with age, eventually leading to biomechanical failure and degeneration of IVD $[15,16]$. There were two historically forms of senescence: one is replicative senescence which is related to shortened telomere length; the other is stress-induced premature senescence which is induced by a variety of environmental stimuli $[17,18]$. Among them, time-dependent accumulation of cell replication and replicative senescence is considered to be more related with aging [19]. Accumulating researches focus on rejuvenating aged NPCs by preventing senescence [11]. However, little progress has been achieved due to the unclear potential regulators or mechanisms of senescence of NPCs. Therefore, elucidating the major regulators and mechanisms underlying NPCs senescence will help us better understand the pathogenesis of IVD aging and degeneration and may illustrate a new therapy target to rejuvenate aged IVD.
Gene microarray technology can simultaneously analyse differences in the expression level of thousands of genes from predefined groups of samples [20]. It also has the advantage of highly effective evaluation of whole genome-wide expression changes [21]. This technology gives researchers a novel point of view to investigate the mechanism of different diseases more deeply. Although the senescence of NPCs plays an important role during IVDD, yet there were limited number of studies that focus on the aberrantly expressed genes during NPC aging.

Thus, the aim of this study was to investigate the abnormally expressed mRNA and signaling pathways during NPC aging by the method of microarray analysis and bioinformatics analysis. We also compared the migration capability between young and old NPCs because decreased migration of IVD cells may be another important reason for the declining regenerative potential of IVD. These analyses may help to elucidate the senescence mechanism of NPCs, which will contribute in identifying the key factors necessary to rejuvenate NPCs in IVDD patients and promoting the effect of cytotherapy in IVDD.

\section{Materials and Methods}

2.1. Ethic Statement. All experimental procedures described blow were reviewed and approved by the Laboratory Animal Ethics Committee of The Sixth Medical Centre of PLA General Hospital, Beijing, China, and carried out in accordance with the relevant guidelines and regulations.

2.2. Isolation and Culture of NPCs. 12 Sprague-Dawley (SD) rats were involved in this experiment and were divided into two groups according to their age: the young group ( $n=6$, 2 months old) and old group ( $n=6,24$ months old). 24month-old rats were defined as the old group according to a previous study [22]. Then, they were sacrificed by intraperitoneal injection of $5 \mathrm{ml} 10 \%$ chloral hydrate. After being soaked in $70 \%$ ethanol for $1 \mathrm{~h}$, the coccygeal NP tissues (C3-C7) of each rat were collected by ophthalmic surgical instruments under a sterile dissecting microscope. After being washed with PBS for three times, the harvested NP tissues were mechanically minced and digested with $0.2 \%$ collagenase II (Sigma-Aldrich, St. Louis, MO, USA) in Dulbecco's modified Eagle's medium-low glucose (DMEM-LG, Solarbio Science \& Technology Co. Ltd., Beijing, China) for $4 \mathrm{~h}$. The suspension was centrifuged at $1000 \mathrm{rpm} / \mathrm{min}$ for $5 \mathrm{~min}$. Then, the suspension solution was discarded, and the pellets were resuspended with standard culture medium containing DMEM-LG, 10\% FBS (Gibco BRL, Grand Island, NY, USA), and $1 \%$ penicillin-streptomycin (Hyclone, USA). Finally, the cell pellets were cultured in $25 \mathrm{~cm}^{2}$ cell culture flasks in a humidified incubator at $37^{\circ} \mathrm{C}$ and $5 \% \mathrm{CO}_{2}$. The culture medium was replaced every two days. When the cells reached $70-80 \%$ confluence, they were collected using $0.25 \%$ trypsinEDTA (Gibco, USA) and subcultured at 1:3; cells at passage 2 were used for experiment.

2.3. Cell Phenotype Identification. To identify the cell phenotype of young and old NPCs, a series of surface markers were 
detected. Both groups of NPCs at P2 were collected and resuspended in cold PBS to a concentration of $1 \times 10^{6}$ cells $/ \mathrm{ml}$. Then, $100 \mu \mathrm{l}$ cell suspension was incubated with antibodies against CD34-PE, CD24-FITC, CD29-PE, CD45-FITC, and CD90-PerCP-Cy5.5 (Abcam, Cambridge, MA, USA) for $30 \mathrm{~min}$ at $4^{\circ} \mathrm{C}$ in the dark. Then, cells were washed twice and resuspended in $500 \mu \mathrm{l}$ PBS. Finally, quantitative analyses for the expression of surface markers of each samples were performed using the FACSCalibur system (FACScan, BD, USA).

\subsection{Senescence-Associated $\beta$-Galactosidase (SA- $\beta$-gal)} Staining. When NPCs were cultured to $80 \%-90 \%$ confluence, SA- $\beta$-gal staining (Beyotime Institute of Biotechnology, China) was performed to analyse the rate of senescent cells in the young and old groups according to the manufacturers' protocol. Briefly, cells were washed by PBS for three times, then fixed with fixative solution for 15 minutes at RT, washed twice with PBS, and stained with X-Gal containing SA- $\beta$-gal working solution overnight at $37^{\circ} \mathrm{C}$ without $\mathrm{CO}_{2}$. Quantification analysis was performed under fluorescence microscopy by determining the average percentage of total SA- $\beta$-gal-positive cells in 5 randomly selected fields of each well.

2.5. Cell Counting Kit-8 Proliferation Assay. To compare the proliferation ability of young and old NPCs, P2 cells were plated at a 96-well plate at 1000 cells/well and cultured in standard medium containing $10 \%$ FBS for 1, 3, 5, 7, and 9 days. At each time point, the medium was replaced by the mixture solution which contains $100 \mu \mathrm{l}$ fresh medium and $10 \mu \mathrm{l}$ CCK- 8 reagent. After being incubated in the humidified incubator at $37^{\circ} \mathrm{C}$ and $5 \% \mathrm{CO}_{2}$ for $4 \mathrm{~h}$, the absorbance of each sample was measured at $450 \mathrm{~nm}$ using a microplate reader (Model 680, Bio-Rad Laboratories K.K., Tokyo, Japan).

2.6. Cell Scratch Assay. Young and old NPCs were seeded in 6-well plates at a concentration of $1 \times 10^{5}$ cells $/ \mathrm{ml}$. After growing to $100 \%$ confluence, parallel scratches were made at the bottom of the 6-well plate with a $200 \mathrm{ml}$ pipet tip. The suspended cells were washed off with serum-free medium, and the width of the scratches was observed and pictured at $0 \mathrm{~h}$ and $24 \mathrm{~h}$ under an Olympus photomicroscope. The migration of the cells was determined by measuring the distance between the wound edges using ImageJ software. Three different areas were measured for each group, and the average distance was calculated for analysis.

2.7. Transwell Assay. The migration capability of young and old NPCs was evaluated with transwell cell culture chambers (pore size $8 \mu \mathrm{m}$, Corning, USA), and these chambers were inserted into 24-well plates. The lower chamber was filled with $600 \mathrm{ml}$ medium with $10 \% \mathrm{FBS}$, and the upper chamber contained $150 \mu \mathrm{l}$ of DMEM along and $5 \times 10^{4}$ cells. After $24 \mathrm{~h}$, the media in both chambers were removed and the nonmigratory cells in the upper chamber were wiped off gently by a cotton swab. After being fixed with $4 \%$ paraformaldehyde, the migrated cells in the lower chamber were stained with $0.1 \%$ crystal violet at room temperature for $30 \mathrm{~min}$. Then, cells traversing the membranes were counted in three randomly selected areas under a light microscope (Olympus Optical Co. Ltd., Tokyo, Japan) at 100x magnification.

2.8. RNA Extraction and Quality Control. After being grown to $90 \%$ confluence, NPCs of the young and old groups were treated with Trizol. Then, the total RNA was extracted and purified with an RNase Kit (Bio-Rad, CA, USA). The quality of derived mRNA was measured by a spectrophotometer (NanoDrop-1000, Thermo Scientific, MA, USA). The mRNA integrity and DNA contamination were detected by agarosegel electrophoresis (results are shown in supplementary materials (available here)).

2.9. Microarray Analysis. Obtained RNA from young and old NPCs was hybridised to Rat GeneChip Clariom ${ }^{\mathrm{TM}} \mathrm{S}$ Array from Affymetrix Corporation. The procedures of hybridization and scanning of the microarray were performed according to Whole Transcript (WT) Expression Arrays User Guide of Affymetrix Corporation. Briefly, after the process of probe set signal integration, background correction, and quantile normalisation, these files were transferred to the Affymetrix Transcriptome Analysis Console software to analyse the differentially expressed genes (DEGs). The threshold set for upand downregulated genes was fold change $\geq 1.5$ and $P<0.05$. The data had been uploaded to the NCBI Gene Expression Omnibus (GEO) and can be accessed via GEO Series accession [GEO:GSE126883] (https://www.ncbi.nlm.nih.gov/geo/ query/acc.cgi?acc=GSE126883).

2.10. GO Functional and KEGG Pathway Enrichment Analysis. The Database for Annotation, Visualization, and Integrated Discovery (DAVID, http://david.abcc.ncifcrf.gov/) is a gene functional classification implement that accommodates a set of functional annotation tools for investigators to analyse the biological roles of genes and to perform GO (Gene Ontology) and KEGG (Kyoto Encyclopedia of Genes and Genomes) pathway enrichment analysis of DEGs. The functions and pathway enrichment of upand downregulated DEGs were analysed using the DAVID database. A count $>2$ and EASE $>0.1$ was considered as the cut-off criteria.

2.11. PPI Network Construction and Module Analysis. Functional PPI analysis was essential to interpret the molecular mechanisms of key cellular activities. The Search Tool for the Retrieval of Interacting Genes (STRING, https://stringdb.org/) database was adopted to obtain the PPI relationships for DEGs. Briefly, DEGs were uploaded to the STRING database, and the result which interaction score is more than 0.7 (high confidence) was visualized in Cytoscape software. Furthermore, significant modules were detected through the MCODE (Molecular Complex Detection) plugin in Cytoscape based on the constructed PPI networks with the criteria of $K$ score $=4$, Degree cut - off $=2$; node score cut - off $=0.2$ , and maximum depth $=100$. GO functional and KEGG pathway enrichment analyses of the highest score module were performed using DAVID.

2.12. Identification of $\mathrm{Hub}$ Genes. Cytoscape software was applied to analyse the hub genes, which are important nodes 
Table 1: Primers used for real-time PCR.

\begin{tabular}{lcc}
\hline Gene name & Forward sequence $5^{\prime}-3^{\prime}$ & ${\text { Reverse sequence } 5^{\prime}-3^{\prime}}^{\prime}$ \\
\hline Fos & CAAACCGACCTACTGTCCC 1 & ACCAACAACCTTGTCGTCATAT \\
Cxcl 1 & GCAACACTTTGTGGCCTGAA & GAGTTGGGACTGGTAGGTGT \\
Igf1 & AAATGGTGAAGGTCGGTGTGAAC & CAACAATCTCCACTTTGCCACTG \\
Ptgs2 & GCACTCTGCTTGCTCACCTTTA & TCCGAATGCTGGAGCCATA \\
Kininogen 1 & TTCGGGAGCACAACAGAGTG & TGAAGTGGTAACCGCTCAGG \\
Periostin & ATGGTCCCGACTGTGAAATGCCAAG & CTATCTCTGGGTAGTCTGCTTTACAC \\
GAPDH & ACAAGCCAACAAAAGGGTTCA & ACGGCCTTCTCTTGATCGC \\
\hline
\end{tabular}

with many interaction partners. We utilized the CytoHubba plugin in Cytoscape to find hub genes and employed six calculation methods: Degree, EPC, EcCentricity, MCC, BottleNeck, and MNC. The intersecting genes derived using these six algorithms represent key candidate genes with important biological regulatory functions. These hub genes were further performed by GO and KEGG pathway enrichment analyses using the DAVID database.

2.13. Real-Time PCR. To validate the microarray results, derived 5 hub genes and the most significantly up- or downregulated genes were selected for the real-time PCR validation. Briefly, complementary DNA (cDNA) was synthesized by the reverse transcript using the Quantscript RT Kit (TianGen Biotech, China) according to the manufacturer's protocol. Then, derived cDNAs were taken for quantitative realtime PCR (qPCR) using a SYBR Premix Ex Taq ${ }^{\mathrm{TM}}$ (Tli RnaseH Plus; TaKaRa Bio, Otsu, Japan) in a Peltier thermal cycler (Bio-Rad Laboratories K.K., Tokyo, Japan) at an ultimate reaction volume of $20 \mu \mathrm{l}$. The cDNA was amplified for 40 cycles. GAPDH was selected as the internal control to calculate the relative expression of target genes by the $2^{-\Delta \Delta C T}$ method. All reactions were performed in triplicate, and the sequences of the used primers are shown in Table 1.

2.14. Statistical Analysis. All the data were expressed as the means \pm SD. The comparative analyses between the groups were made by independent sample $t$-tests to determine the significant difference through SPSS 20.0 software (Chicago IL, USA). A chi-square test was adopted for enumeration data. $P<0.05$ was considered statistically significant.

\section{Results}

3.1. Immunophenotypes of Young and Old NPCs. NPCs were successfully isolated from rat nucleus pulposus tissues. The P2 generation of young NPCs exhibited a characteristic of small, spindle-like morphology, with an abundant cytoplasm containing large ovoid and prominent nucleoli. Old NPCs had a more relatively round shape with polygonal and flat morphology (Figure 1(a)).

A series of cell surface antigens were selected to detect the cell surface marker of young and old NPCs [23, 24]. Both groups of NPCs were highly positive for CD29 and CD90 and $\mathrm{CD} 24$ (Figures 1(b) and 1(c)). However, the expression of CD24 was significantly lower in the old group than that in the young group $(P<0.05)$. The positive rates in the young group were CD29 $(99.15 \pm 0.67 \%)$, CD90 $(97.63 \pm 0.62 \%)$, and CD24 (92.68 $\pm 0.88 \%)$. The positive rates in the old group were CD29 $(97.3 \pm 1.26 \%)$, CD90 (96.36 $\pm 0.54 \%)$, and CD24 (77.91 $\pm 2.49 \%)$. All NPCs were negative for the hematopoietic stem cell markers CD34 $(1.1 \pm 0.4 \%$ in the young group and $3.98 \pm 0.22 \%$ in the old group) and CD 45 $(3.98 \pm 0.22 \%$ in the young group and $1.9 \pm 0.16 \%$ in the old group) (Figures 1(b) and 1(c)).

3.2. $\beta$-Galactosidase (SA- $\beta$-gal) Staining and Aging-Related Decline in Proliferation and Migration Ability. $\beta$-Galactosidase (SA- $\beta$-gal) staining is a sensitive measurement to detect senescent cells, the results showed a higher positive staining cell percentage in the old group $(45.32 \pm 6.87 \%)$ than that in the young group $(10.84 \pm 1.41 \%)(P<0.05)$ (Figures $2(\mathrm{a})$ and 2(b)). To assess the proliferation of young and old NPCs, CCK-8 assay was performed. Results showed old NPCs went into early plateau phase approx. 7 days after initially culture and young NPCs did not enter a growth plateau until 9 days of culture (Figure 2(c)). The migration ability was assessed through transwell assay and cell scratch assay. Results of transwell assay showed a decreased migration cell number in the old group $(56.33 \pm 8.327)$ compared with the young group cells $(169.3 \pm 16.44)(P<0.05)$ (Figures $2(\mathrm{e})$ and $2(\mathrm{f}))$. Quantification of the migration area percent after $24 \mathrm{~h}$ of the young group $(41.02 \pm 6.13 \%)$ is higher than that of the old group $(14.9 \pm 3.15 \%)(P<0.05)$, indicating a decreased migration speed of old NPCs (Figures 2(d) and 2(g)). Thus, our results clearly demonstrate a dramatic decrease in the proliferous and migratory capacity of NPCs during aging.

3.3. Identification of Differentially Expressed Genes. To detect molecular factors involved in NPC aging, we performed microchip hybridization with RNA from NPCs of the young and old groups. A total of 1038 differentially expressed genes (DEGs) were detected, including 617 upregulated genes and 421 downregulated genes. The upregulated genes refer to those increase expressed in old NPCs. Volcano plot was plotted to show the DEGs of two groups according to the gene expression values (Figure 3). The hierarchical clustering heat map is shown in the supplementary materials. The greatest upregulated gene is kininogen 1 (fold change $=25.06$ ), followed by lipocalin 2, upregulated, and EGF-containing fibulinlike extracellular matrix protein 1 , upregulated. The greatest downregulated gene is periostin (fold change $=-24.05$ ), 

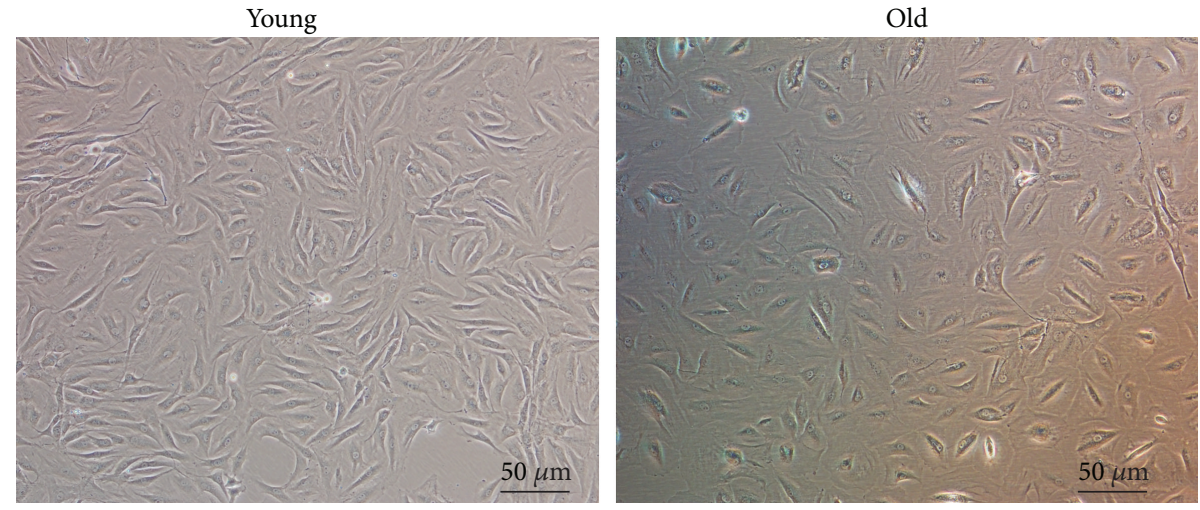

(a)
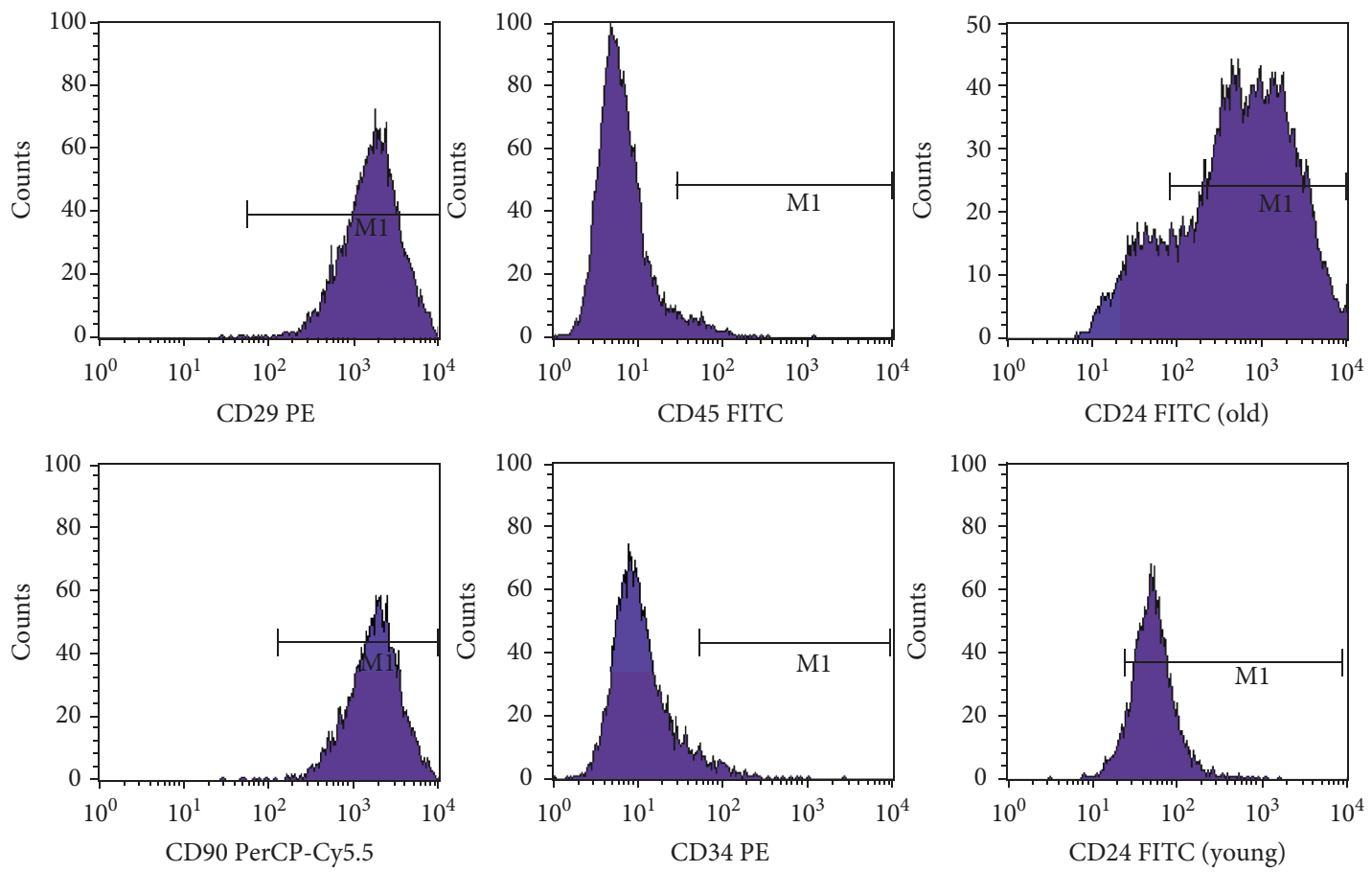

(b)

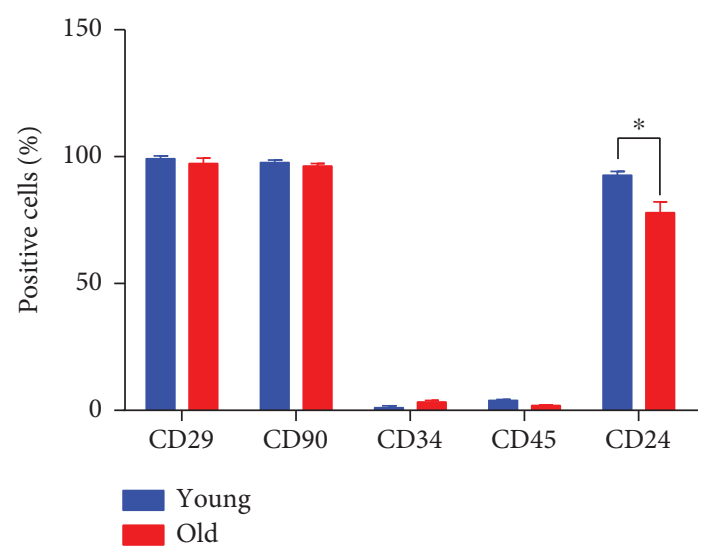

(c)

FIGURE 1: The cell morphology and immunophenotype identification of young and old NPCs. (a) Cell morphology of P2 young and old NPCs. (b, c) Both young and old NPCs exhibited high expression of CD29 and CD90 (>95\%) and low expression of CD34 and CD45 $(<5 \%)$. The expression of CD24 in the young group was $92.68 \pm 0.88 \%$ and in the old group was $77.91 \pm 2.49 \%$. The difference of CD24 expression between young and old NPCs was significant $(P<0.05) .{ }^{*} P<0.05$ compared with the young group. 


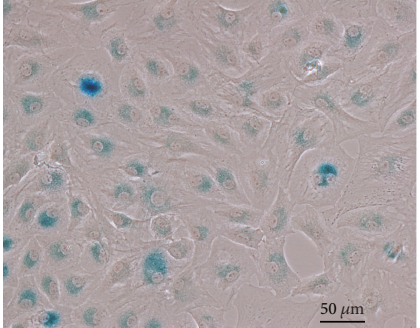

Young

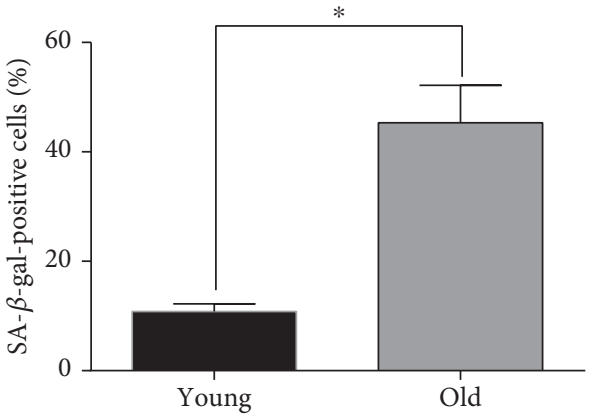

(b)

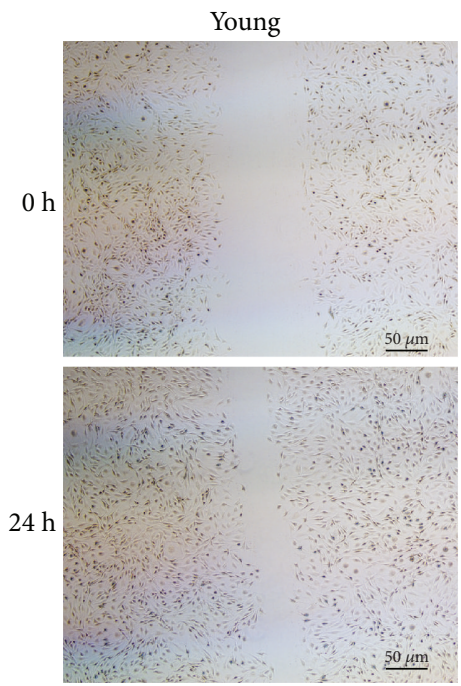

Young

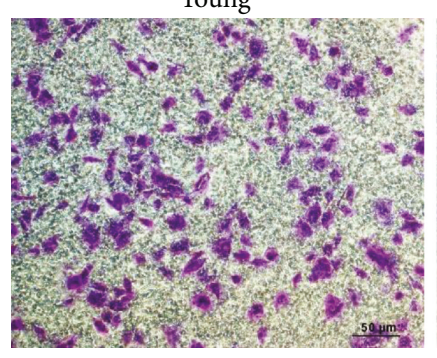

Old

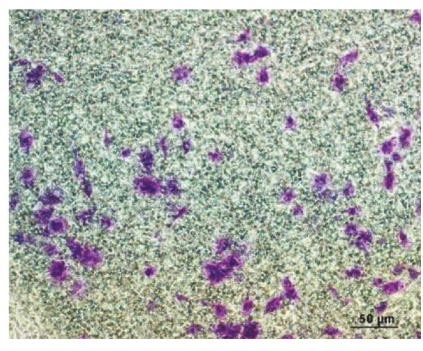

(e) (d)

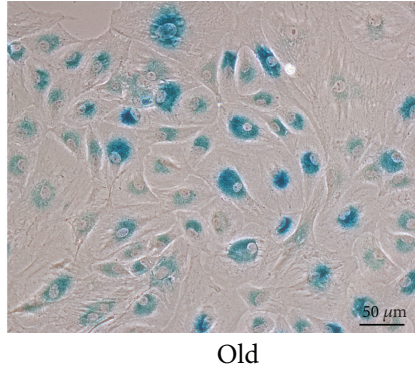

(a)

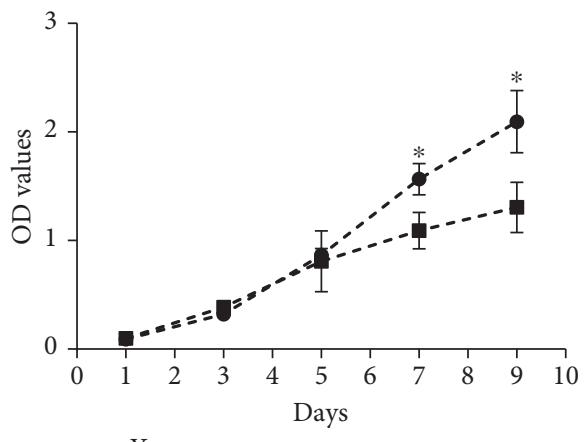

-- Young

(c)
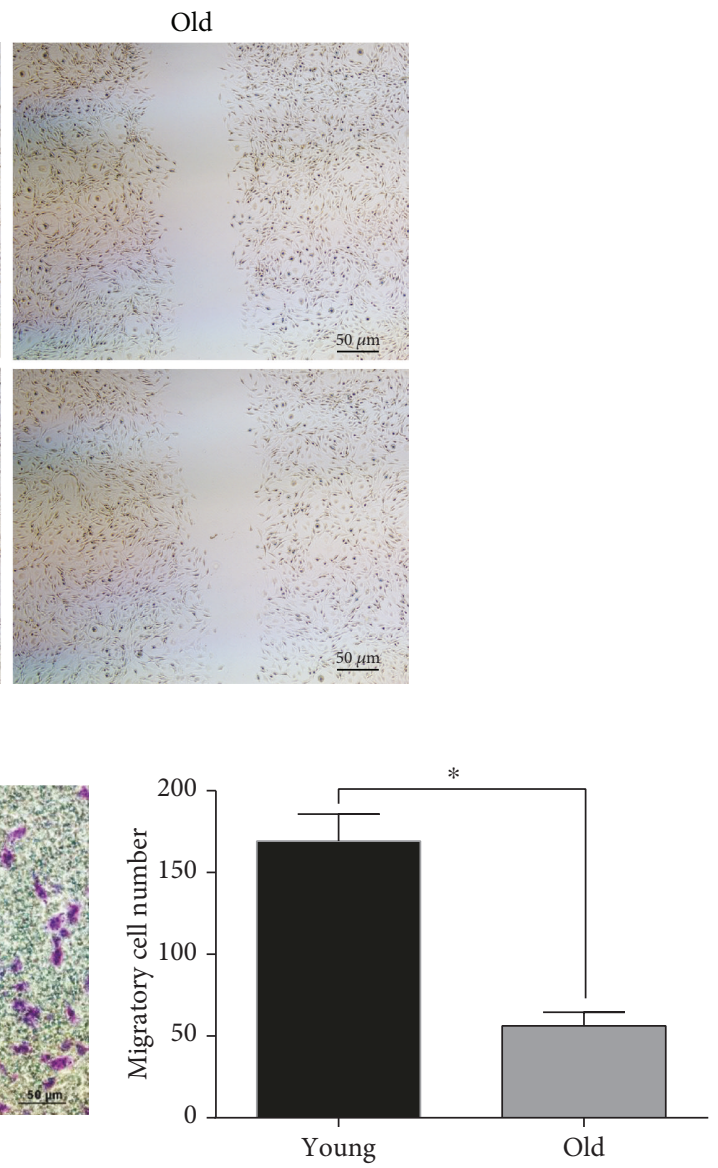

(f)

Figure 2: Continued. 


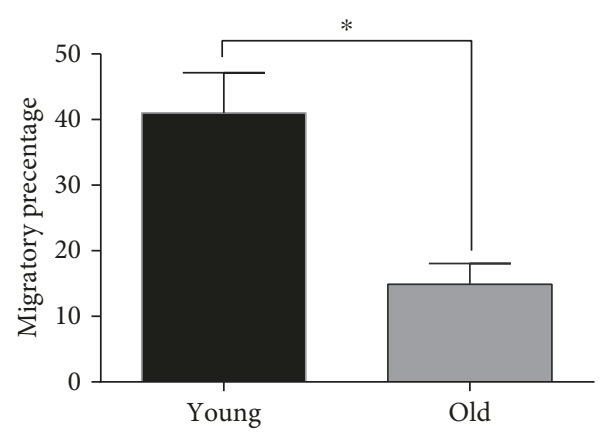

(g)

FIGURE 2: The aging phenotype and migration capacity of young and old NPCs. $(a, b)$ Representative images of SA- $\beta$-gal staining for the detection of senescent NPCs from the young and old groups. Quantitative analysis showed that the senescent cell number in the old group was higher than that in the young group. (c) Cell proliferation curve detected by CCK- 8 assay. The OD value is higher in the young group after 7 days culture, indicating a higher proliferation capability of young NPCs. (d, g) Cell scratch assay showed declined migrated percentage in old NPCs after $12 \mathrm{~h}$. (e, f) Transwell assay showed a declined migrated cell number in the old group. All samples examined in triplicate. Data are presented as the mean \pm SD. ${ }^{*} P<0.05$.

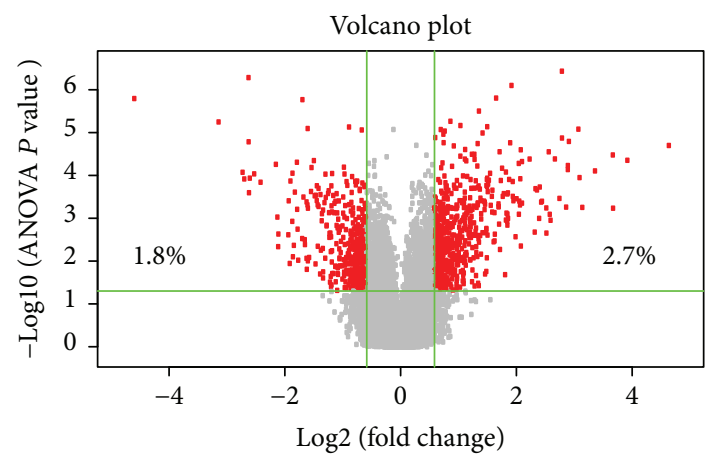

FIGURE 3: Volcano plot showed differentially expressed genes between the young and old groups. Volcano plot of differentially expressed genes in young and old group. Left: significantly downregulated genes, which account for $1.8 \%$ of total detected probes. Middle: nondifferentially expressed genes. Right: significantly upregulated genes, which account for $2.7 \%$ of total detected probes (based on $|\log 2 \mathrm{FC}|>2$ and $P<0.05$ ). Heat map and hierarchical clustering of DEG profile comparison between the young and old NPCs are included in the supplementary materials.

followed by neuronal regeneration-related protein, downregulated, and dermatopontin, downregulated. The top 10 up- and downregulated genes are shown in Tables 2 and 3.

\subsection{GO Functional and KEGG Pathway Enrichment Analysis} of DEGs. The functions of up and downregulated DEGs were evaluated at DAVID. The GO analysis showed that, in terms of biological processes (BP), the upregulated genes were mainly enriched in response to lipopolysaccharide and response to organic cyclic compound and negative regulation of cell proliferation (Table 4 and Figure 4). The downregulated genes were mainly enriched in cell adhesion, endodermal cell differentiation, and ossification (Table 5 and Figure 5). In terms of cellular components (CC), upregulated genes were mainly enriched in the extracellular space and extracellular exosome (Table 4 and Figure 4). Downregulated genes were mainly enriched in the extracellular matrix and basement membrane (Table 5 and Figure 5). In terms
TABLE 2: The top 10 upregulated genes.

\begin{tabular}{lcc}
\hline Gene names & Fold change & $P$ value \\
\hline Kininogen 1 & 25.06 & $2.00117 E-05$ \\
Lipocalin 2 & 15.25 & $4.43512 E-05$ \\
EGF-containing fibulin-like & 12.85 & 0.000583062 \\
extracellular matrix protein 1 & 12.81 & $3.332 E-05$ \\
Collagen 14a1 & 10.35 & $7.85477 E-05$ \\
Phospholipase A2 & 8.89 & 0.000557976 \\
Interferon-induced & 8.48 & $8.31466 E-06$ \\
transmembrane protein 1 & 7.57 & $1.60859 E-05$ \\
LOC100125362 & 7.48 & $5.87991 E-05$ \\
Carboxypeptidase X & 7.48 & 0.000073019 \\
Complement component 6 & & \\
Inhibitor of DNA binding 1 & &
\end{tabular}

Filter criteria for significant difference gene: $\mid$ fold change $\mid>1.5, P$ value $<0.05$.

TABLE 3: The top 10 downregulated genes.

\begin{tabular}{lcc}
\hline Gene names & Fold change & $P$ value \\
\hline Periostin & -24.05 & $1.60411 E-06$ \\
Neuronal regeneration-related protein & -8.77 & $5.64411 E-06$ \\
Dermatopontin & -6.57 & $8.44669 E-05$ \\
Integrin alpha 4 & -6.44 & 0.000120932 \\
Syntaxin-binding protein 5-like & -6.12 & $5.20189 E-07$ \\
Fibulin 2 & -6.11 & $1.64021 E-05$ \\
Dihydropyrimidinase-like 3 & -6.09 & 0.000253521 \\
Calponin 1 & -6.03 & 0.000115957 \\
Sphingosine-1-phosphate receptor 3 & -5.72 & $9.20555 E-05$ \\
Gliomedin & -5.30 & 0.000144277 \\
\hline
\end{tabular}

Filter criteria for significant difference gene: $\mid$ fold change $\mid>1.5, P$ value $<0.05$.

of molecular function (MF), upregulated genes were mainly enriched in endopeptidase inhibitor activity and transcriptional activator activity (Table 4 and Figure 4), while 
TABLE 4: List of top 5 terms of upregulated genes enriched by GO and KEGG analysis.

\begin{tabular}{|c|c|c|}
\hline Terms & Count & $\begin{array}{c}-\log 10 \\
(P \text { value })\end{array}$ \\
\hline KEGG:rno04668:TNFsignaling pathway & 15 & 4.863619311 \\
\hline $\begin{array}{l}\text { KEGG:rno04151:PI3K-Aktsignaling } \\
\text { pathway }\end{array}$ & 27 & 4.249211392 \\
\hline KEGG:rno05200:Pathways in cancer & 29 & 3.912805981 \\
\hline $\begin{array}{l}\text { KEGG:rno04630:Jak-STAT signaling } \\
\text { pathway }\end{array}$ & 14 & 3.272521081 \\
\hline KEGG:rno04512:ECM-receptor interaction & 10 & 2.566175661 \\
\hline $\begin{array}{l}\text { GO BP:0032496 response to } \\
\text { lipopolysaccharide }\end{array}$ & 29 & 7.485884419 \\
\hline $\begin{array}{l}\text { GO BP:0014070 response to organic } \\
\text { cyclic compound }\end{array}$ & 28 & 7.172760304 \\
\hline $\begin{array}{l}\text { GO BP:0008285 negative regulation } \\
\text { of cell proliferation }\end{array}$ & 33 & 6.863888026 \\
\hline GO BP:0001666 response to hypoxia & 27 & 6.668325051 \\
\hline $\begin{array}{l}\text { GO BP:0050680 negative regulation } \\
\text { of epithelial cell proliferation }\end{array}$ & 13 & 5.878814283 \\
\hline $\begin{array}{l}\text { GO MF:0004866 endopeptidase } \\
\text { inhibitor activity }\end{array}$ & 9 & 5.741812795 \\
\hline $\begin{array}{l}\text { GO MF:0001077 transcriptional activator } \\
\text { activity, RNA polymerase II } \\
\text { core promoter proximal region } \\
\text { sequence-specific binding }\end{array}$ & 24 & 5.695518301 \\
\hline GO MF:0005509 calcium ion binding & 39 & 3.672674033 \\
\hline $\begin{array}{l}\text { GO MF:0008134 transcription factor } \\
\text { binding }\end{array}$ & 23 & 3.591418924 \\
\hline $\begin{array}{l}\text { GO MF:0043565 sequence-specific } \\
\text { DNA binding }\end{array}$ & 33 & 3.418128712 \\
\hline GO CC:0005615 extracellular space & 99 & 16.94638876 \\
\hline GO CC:0070062 extracellular exosome & 135 & 9.756235253 \\
\hline GO CC:0009986 cell surface & 48 & 8.51199254 \\
\hline $\begin{array}{l}\text { GO CC:0005578 proteinaceous } \\
\text { extracellular matrix }\end{array}$ & 25 & 6.200499673 \\
\hline GO CC:0031012 extracellular matrix & 25 & 6.169491531 \\
\hline
\end{tabular}

downregulated genes were mainly enriched in extracellular matrix structural constituent and calcium ion binding (Table 5 and Figure 5).

The KEGG pathway analysis showed that the upregulated genes were enriched in 36 pathways; the most significant pathway was the TNF signaling pathway, and the top 5 significant pathways are shown in Table 4 and Figure 4 . The downregulated genes were enriched in 19 pathways; among them, the most significant pathway is ECM-receptor interaction, and the top 5 significant pathways are shown in Table 5 and Figure 5.

3.5. PPI Analysis and Hub Gene Screening. Based on information from the STRING database, a PPI network comprising 311 nodes and 696 edges with parameters including a minimum required interaction score $>0.7$ (high confidence) was constructed using the Cytoscape software (Figure 6(a)). Then, the networks were analysed by plugin MCODE with the criteria of node score $>4$ and number of nodes $>4$. Finally, 3 significant modules were selected (Figures 6(b)6(d)). The KEGG pathways enriched of the genes in the highest scored modules (score 6.276) were the TNF signaling pathway, PI3K-Akt signaling pathway, and cytokinecytokine receptor interaction; the GO biological processes were chiefly enriched in cellular response to organic substance, skeletal muscle cell differentiation, and inflammatory response (Table 6). Then, we detected the hub genes in the network. After running the CytoHubba plugin, there were 5 hub genes identified by the 6 calculation methods (Degree, EPC, EcCentricity, BottleNeck, MCC, and MNC). Results are listed in Table 7. The 6 most significant genes were chemokine (C-X-C motif), ligand 1 (Cxcl1), early growth response 1 (Egr1), FBJ osteosarcoma oncogene (Fos), insulin-like growth factor 1 (Igf1), and prostaglandin-endoperoxide synthase 2 (Ptgs2). Furthermore, we performed GO and KEGG enrichment analysis of hub genes in DAVID. These hub genes were mainly enriched in the TNF signaling pathway and pathways in cancer (Table 8). The chief GO biological processes were in response to lipopolysaccharide and response to glucocorticoid (Table 8).

3.6. Validation of Hub Gene by Real-Time PCR. To validate the results of microarray date, we selected the 5 hub genes and the most significantly up- or downregulated genes for real-time PCR analysis. Results were showed consistent with the microarray data. The gene chip analysis demonstrated these mRNAs were upregulated up to 2.643-fold (CXCL1), 5.429-fold (Fos), 2.386-fold (Igf1), 2.369-fold (Egf1), and 3.748-fold (Ptgs2). The RT-PCR results exhibit the expression of CXCL1 $(P<0.05)$, Fos $(P<0.05)$, Igf1 $(P<0.05)$, Egf1 $(P<0.05)$, and Ptgs2 $(P<0.05)$ in the old group which were significantly increased up to 3.8 -fold, 4.36 -fold, 2.39 fold, 3.04-fold, and 3.27-fold, respectively. The expression of periostin $(P<0.05)$ in the old group was -12.7 -fold compared with that in the young group, and the expression of kininogen $1(P<0.05)$ in the old group was 12.4 -fold compared with that in the young group. Results of RT-PCR were consistent with the results of microarray data. The results are shown in Figure 7.

\section{Discussion}

Cellular senescence serves as an important disease-causing determinant $[12,17]$. Senescence of NPCs with age is closely related to the change of IVD aging and degeneration $[7,10$, 11]. In this study, we compared the biological functions and analysed the differentially expressed gene in young and old NPCs to uncover the potential therapeutic target during NPC senescence. This study has a significant value in clinic therapy of IVDD because the key regulated genes during the NPC senescence could be manipulated to reactivate the senescent NPCs. It would be unnecessary to isolate NPCs from the tissue by invasive operation, patients may just need to receive molecules to rejuvenate NPCs, and then, the selfrepair procedure could be started.

To identify the surface phenotypes of isolated young and old NPCs, a variety of surface markers were adopted for 


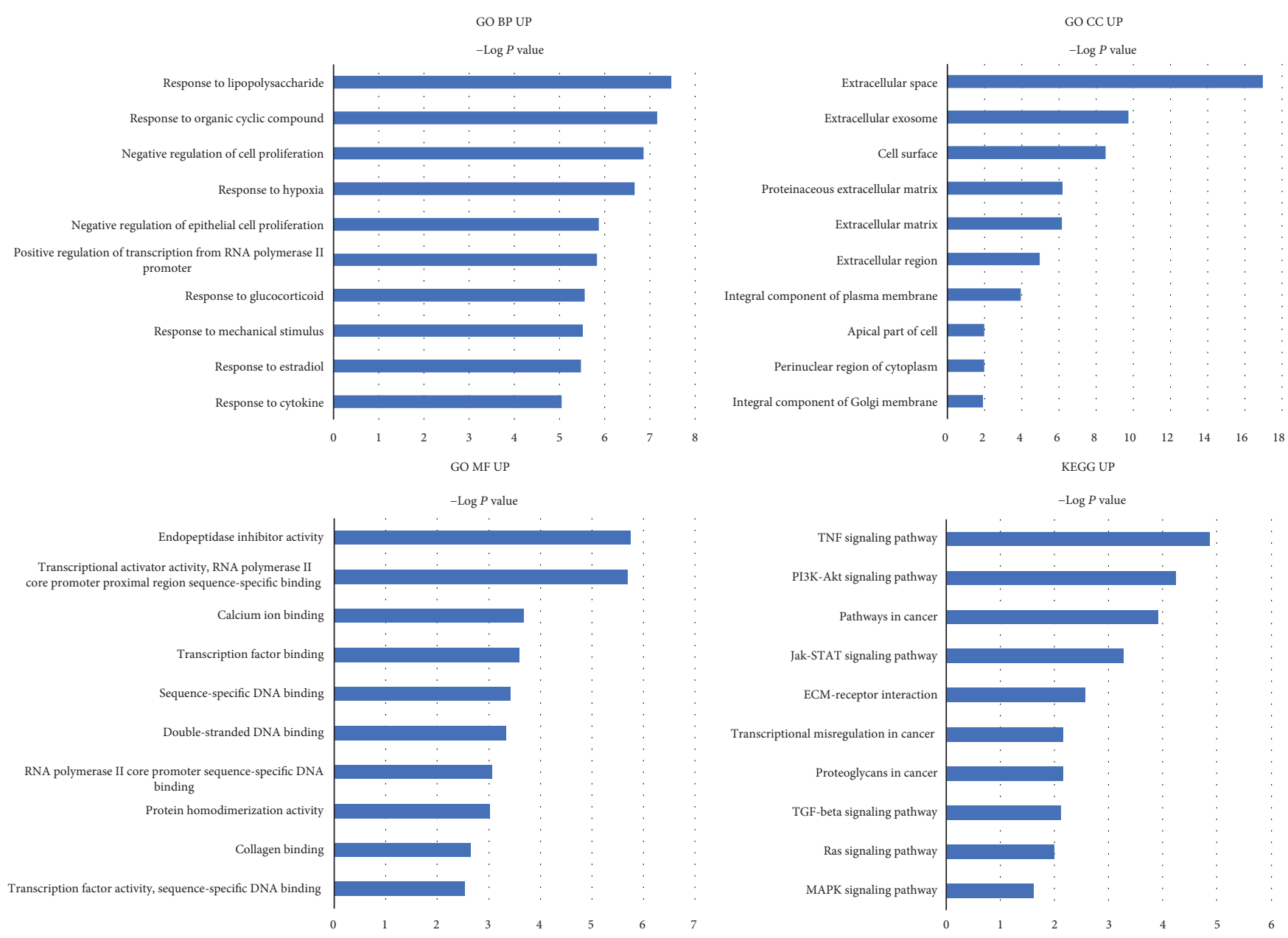

FIGURE 4: Go function and KEGG pathway enrichment analysis of upregulated genes. Top 5 most significantly enriched GO terms and KEGG pathways of upregulated genes. The length of the bars on the $x$-axis represents the negative logarithm of the $P$ value (-log $10 P)$ of each pathway; higher $(-\log 10 P)$ values indicate a higher significance, and lower $(-\log 10 P)$ values indicate a lower significance. The GO terms and KEGG pathway names are shown on the $y$-axis. GO MF UP, GO CC UP, and GO BP UP refer to GO terms enriched by upregulated genes. KEGG UP refers to the KEGG terms enriched by upregulated genes.

detection according to previous studies [23-25]. CD24 is a glycosylphosphatidylinositol-anchored cell surface protein, which has been defined as a marker of healthy NPCs by Risbud et al. [23]. Tang et al. found a strong expression of CD24 in juvenile human NP tissue, and its expression would decline with age [26]. Although the species in this study was different with previous studies, we also found the expression of CD24 in old NPCs decreased significantly than that in young NPCs. This result indicated that CD24 may serve as a marker of NPC senescence. We further detected the expression of CD90, which is a cell-surface-anchored glycoprotein found in many kinds of stem/progenitor cells [27]. In the present study, CD90 was highly expressed in both young and old NPCs. However, Tang et al. found that CD90 is only expressed in AF cells and may serve as a non-NP marker in rats [14]. We considered that this may be due to the different isolation method used in their study. Previous study of Molinos et al. found that NPCs highly express CD29 and Brachyury with a low expression of CD34, CD45, and CD146 [24]. In our study, the expression of CD34 and CD45 was lower than $5 \%$ in both the young and old groups, which indicated no contamination of hematopoietic-lineage cells. Both young and old NPCs could highly express CD29 (>95\%), and its expression discovered no difference in young and old NPCs, which was consistent with the previous study [24].

Then, we compared the proliferation and migration capabilities of young and old NPCs. An age-related decline in the growth kinetics was reported for NPCs before. Jeong et al. found that human NPCs from young patients have a higher proliferation ability and less SA- $\beta$-gal staining percentage than old ones [28]. In line with the abovementioned research, our study also showed a diminished proliferation capacity of old NPCs. We further investigate the migration capability between young and old NPCs, and results showed a declined migration ability in old NPCs than young NPCs. Thus, based on the combined above results, we propose that the declined proliferous and migratory capabilities of NPCs are two key processes of IVD aging.

In the present study, we further revealed the DEGs between young and old NPCs. Among the downregulated genes, periostin was the most significantly altered gene. Previous studies have shown that periostin could regulate the 
TABLE 5: List of top 5 terms of down regulated genes enriched by GO and KEGG analysis.

\begin{tabular}{|c|c|c|}
\hline Terms & Count & $\begin{array}{l}-\log 10 \\
(P \text { value })\end{array}$ \\
\hline KEGG:rno04512:ECM-receptor interaction & 12 & 6.305467082 \\
\hline KEGG:rno04510:Focal adhesion & 16 & 5.283730936 \\
\hline $\begin{array}{l}\text { KEGG:rno04151:PI3K-Akt signaling } \\
\text { pathway }\end{array}$ & 18 & 3.918980827 \\
\hline $\begin{array}{l}\text { KEGG:rno04974:Protein digestion and } \\
\text { absorption }\end{array}$ & 8 & 2.964674869 \\
\hline KEGG:rno04024:cAMP signaling pathway & 11 & 2.565239027 \\
\hline GO BP:0007155 cell adhesion & 19 & 5.677119186 \\
\hline $\begin{array}{l}\text { GO BP:0035987 endodermal cell } \\
\text { differentiation }\end{array}$ & 6 & 3.820807813 \\
\hline GO BP:0001503 ossification & 9 & 3.307311242 \\
\hline GO BP:0007160 cell-matrix adhesion & 8 & 3.272206365 \\
\hline $\begin{array}{l}\text { GO BP:0010628 positive regulation } \\
\text { of gene expression }\end{array}$ & 17 & 2.767181699 \\
\hline $\begin{array}{l}\text { GO MF:0005201 extracellular matrix } \\
\text { structural constituent }\end{array}$ & 7 & 3.709602001 \\
\hline GO MF:0005509 calcium ion binding & 28 & 3.596212048 \\
\hline $\begin{array}{l}\text { GO MF:0003735 structural constituent } \\
\text { of ribosome }\end{array}$ & 18 & 2.633105261 \\
\hline GO MF:0044325 ion channel binding & 9 & 2.592276912 \\
\hline GO MF:0005178 integrin binding & 8 & 2.584636716 \\
\hline GO CC:0031012 extracellular matrix & 28 & 12.71649934 \\
\hline GO CC:0005604 basement membrane & 16 & 9.863156842 \\
\hline GO CC:0005615 extracellular space & 53 & 6.939058168 \\
\hline $\begin{array}{l}\text { GO CC: } 0005578 \sim \text { proteinaceous } \\
\text { extracellular matrix }\end{array}$ & 20 & 6.645158563 \\
\hline GO CC:0005925 focal adhesion & 25 & 6.484468766 \\
\hline
\end{tabular}

proliferation and differentiation of many kinds of cells such as periodontal ligament mesenchymal stem cells $[29,30]$, skeletal stem cells [31], and adipose-derived stem cell [32, 33]. Furthermore, periostin could interact with structural collagens, thereby influencing the mechanical structure of the ECM in a local tissue [34]. Egbert et al. found that periostin contributes to proper collagen function and is downregulated during skin aging, indicating an important role of periostin in the regulation of collagen function [35]. Previous research found that periostin is also being expressed in the human and rat IVD and has relevance to the IVDD because it binds to several ECM components such as fibronectin, tenascin, and collagen $\mathrm{V}$ and is related to the expression of several inflammatory cytokines such as IL- 4 and TNF- $\alpha$ [36]. However, Tsai et.al reported an increased expression of periostin in IVD cells during IVDD, which was contradicting with our results [37]. We consider that it is because their model represents rapid injury of IVD, thus stimulating periostin expression to regulate the structure of ECM. The reaction of cells to the change of environment might be declined with age, thus showing a downregulation of periostin of NPCs. Consistent with our hypothesis, Graja et.al reported that loss of periostin occurs in aging adipose tissue is closely associated with the age-related alterations of the adipose tissue extracellular matrix [32]. Moreover, Duchamp de Lageneste et.al discovered that the bone regenerative potential of skeletal stem cells in periosteum is determined by periostin [31]. Thus, methods that upregulate the expression of periostin might reverse the malfunction of NPCs during aging. Among the high expression genes, kininogen 1 was the highest one. Kininogen 1 has been detected in numerous pathophysiological conditions, such as arthritis [38] and inflammatory bowel disease [39]. Although there is no literature that reported the association of kininogen 1 with IVD aging, the relationship of kininogen 1 with aging in other tissues has been recognized [40, 41]. Besides, Dai et.al reported that cleaved kininogen could accelerate the onset of endothelial progenitor cell senescence by activating the ROS-p38 kinase-p16INK4a signaling cascade [42]. Therefore, kininogen 1 might have a similar effect on NPC senescence.

Functional annotation of upregulated genes was conducted by GO and KEGG pathway analysis. Interestingly, the top 5 enrichments of GO BP included response to lipopolysaccharide (GO BP:003249), negative regulation of cell proliferation (GO BP:0008285), and response to hypoxia (GO BP:0001666). Lots of literatures reported that lipopolysaccharide could induce inflammatory response of IVD; thus, the biological processes in response to lipopolysaccharide indicated an important role of inflammation in NPC senescence [43]. Besides, hypoxia and inflammation are two important characteristics of the environment of degenerated IVD [44]; thus, these results highlighted a vital role of a local environment in NPC senescence. Additionally, the most significant signaling pathways of upregulated genes included the TNF signaling pathway (KEGG:rno04668), PI3K-Akt signaling pathway (KEGG:rno04151), and pathways in cancer (KEGG:rno05200). The TNF signaling pathway has been widely recognized as a vital regulator of the inflammatory cascade during IVDD $[45,46]$. Thus, our results showed that the biological function of old NPCs might been influenced by the harsh conditions existing in the microenvironment of IVD. Among the downregulated genes, GO functional enrichment of BP is mainly enriched at cell adhesion (GO BP:0007155), cell-matrix adhesion (GO BP:0007160), and endodermal cell differentiation (GO BP:0035987). Pathway enrichment analysis is mainly at ECM-receptor interaction (KEGG:rno04512) and focal adhesion (KEGG:rno04510). Interestingly, these results were consistent with our cellular experiment results which showed a declined migratory capability of old NPCs. Therefore, these results indicated a decreased migratory capability of old NPCs.

We further analysed the hub genes in the PPI network by 6 calculation methods. All derived 5 hub genes (Cxcl1, Egr1, Ptgs2, Fos, and Igf1) were upregulated in the old group. We further conducted real-time PCR to verify the results of microarray. Results showed a consistent expression trend with microarray. Cxcl1, Egr1, and Ptgs2 have been reported to be existing in IVD tissues and revealed a close relationship with the immune response and inflammation. [47-49] Fos is a well-studied oncogene. It was reported that Fos existed in the herniated disc tissue instead of the healthy disc [50]. Besides, a recent study had shown that Fos could regulate the transcription of Sox 9 and that the cfos-Sox9 axis is critical 

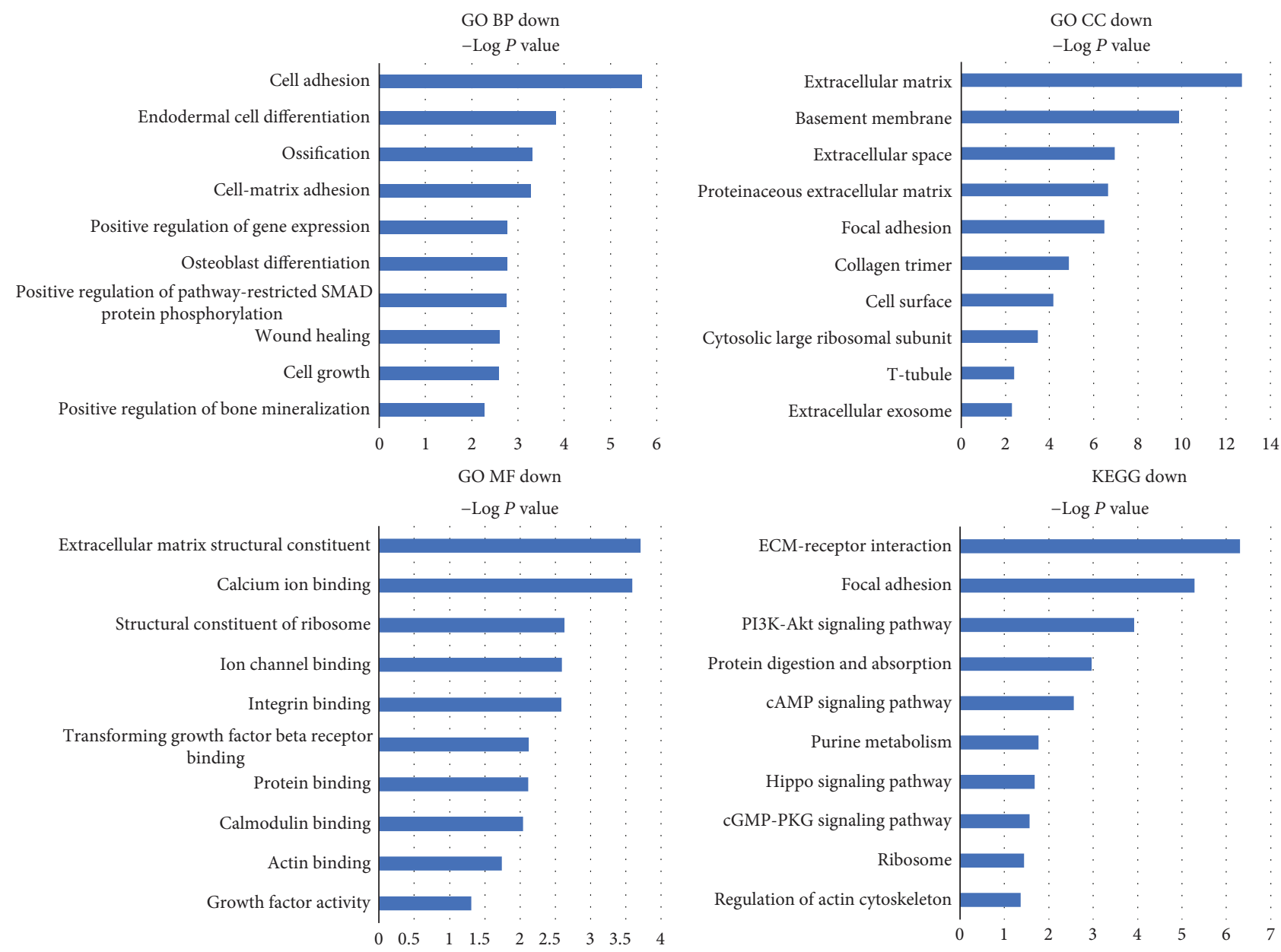

FIGURE 5: Go functional and KEGG pathway enrichment analysis of downregulated genes. Top 5 most significantly enriched GO terms and KEGG pathways of downregulated genes. The length of the bars on the $x$-axis represents the negative $\operatorname{logarithm}$ of the $P$ value $(-\log 10 P)$ of each pathway; higher $(-\log 10 P)$ values indicate a higher significance, and lower $(-\log 10 P)$ values indicate a lower significance. The GO terms and KEGG pathway names are shown on the $y$-axis. GO MF DOWN, GO CC DOWN, and GO BP DOWN refer to GO terms enriched by downregulated genes. KEGG DOWN refers to the KEGG terms enriched by downregulated genes.

for the role of cfos in the induction of chondroblastic Osteosarcoma [51]. Considering that SOX-9 is also an important marker of nucleus pulposus cells, the role of cfos in the regulation of NPCs during aging is needed to be deeply investigated. Igf1 is a growth factor known to activate matrix metabolism. Igf1 has also been found to have a close relationship with IVDD [52]. A previous study has described that the responsiveness of chondrocytes to IGF-I decreased with age [53]. Okuda et.al found that the increased expression of IGF-I binding proteins (IGFBPs) and downregulation of IGF-I receptor might be the two key mechanisms for the aging-related nonresponsiveness to IGF-1 [54]. Therefore, we hypothesized the upregulated expression of IGF-1 might be the compensation feedback in old NPCs. We also performed the GO and KEGG analysis of derived hub genes. Results showed that the TNF signaling pathways in response to lipopolysaccharide were enriched, which is consistent with the GO and KEGG results of upregulated genes.

The three most significant submodules of DEGs were extracted from the PPI network with MCODE scores of $\geq 4$.
After GO functional and KEGG pathway enrichment analyses of the DEGs in the highest scored modules, the genes in this module were mainly associated with the cellular response to organic substance (GO:0071310), skeletal muscle cell differentiation (GO:0035914), and inflammatory response (GO:0006954). The pathways were enriched in the TNF signaling pathway (rno04668), and PI3K-Akt signaling pathway (rno04151), which further illustrated the importance of these two pathways during NPC aging.

There were several limitations in this study. First, numerous RNA probes were detected in the microarray analysis and this limited the validation of the gene chip results. Therefore, we only interpreted the results based on previous studies and our interests. It was reported that NP consists of a mixture of small chondrocyte-like mesenchymal cells and larger notochordal-derived cells [26]. In mature NP tissues, notochordal cells gradually disappeared, replaced by smaller fibrochondrocyte-like cells [8]. However, the harvested cells in this study were mainly fibrochondrocyte-like cells in both the young and old groups, which was different with the 


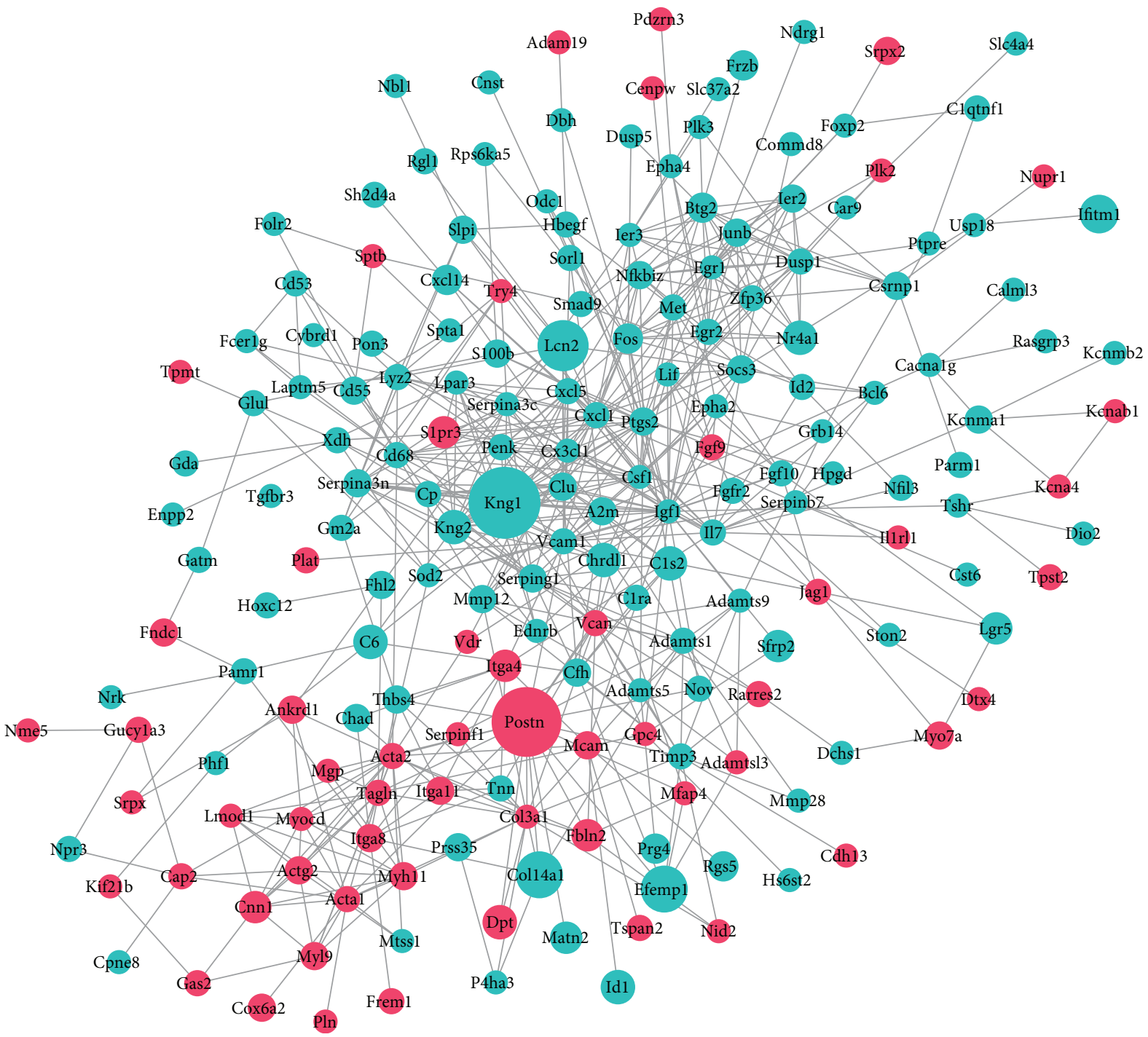

(a)

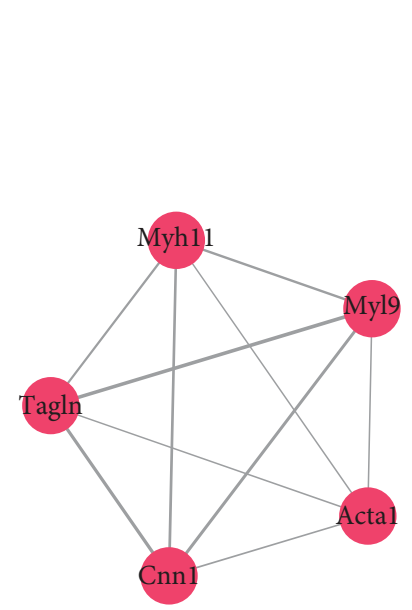

(b)

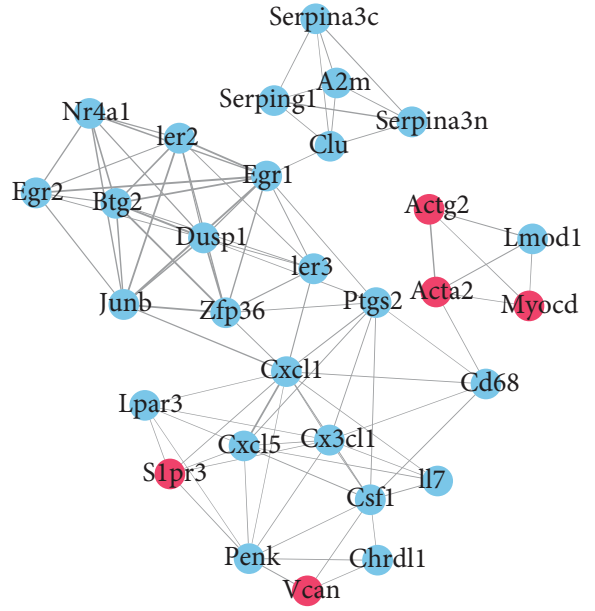

(c)

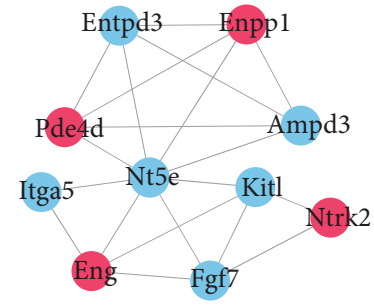

(d)

FIGURE 6: Protein-protein interaction network and significant modules of differentially expressed genes. (a) Red: significantly downregulated genes; green and light blue: significantly upregulated genes. Node size is positively related to fold change; edge width is positively related to the combined score. (b, c, d) Significant modules in the protein-protein interaction (PPI) network with a MCODE score $\geq 4$ : (b) module 1 (score $=5)$; (c) module 2 (score $=6.276)$; and $(\mathrm{d})$ module 3 ( score $=4.444)$. 
TABLE 6: KEGG and GO analysis of the highest scored module genes.

\begin{tabular}{lcc}
\hline Terms & $\begin{array}{c}-\log 10 \\
(P \text { value })\end{array}$ & Count \\
\hline $\begin{array}{l}\text { GO BP:0071310 cellular response to } \\
\text { organic substance }\end{array}$ & 4.428041285 & 4 \\
$\begin{array}{l}\text { GO BP:0035914 skeletal muscle cell } \\
\text { differentiation }\end{array}$ & 4.06731104 & 4 \\
$\begin{array}{l}\text { GO BP:0006954 inflammatory response } \\
\text { GO BP:0009611 response to wounding }\end{array}$ & 4.016795968 & 6 \\
$\begin{array}{l}\text { GO BP:0032496 response to } \\
\text { lipopolysaccharide }\end{array}$ & 3.016316347 & 5 \\
$\begin{array}{l}\text { KEGG:rno04668:TNF signaling pathway } \\
\text { KEGG:rno04151:PI3K-Akt signaling }\end{array}$ & 3.913584453 & 5 \\
$\begin{array}{l}\text { pathway } \\
\begin{array}{l}\text { KEGG: rno04060:Cytokine-cytokine } \\
\text { receptor interaction }\end{array}\end{array}$ & 1.320427527 & 4 \\
\hline
\end{tabular}

TABLE 7: The hub genes were analysed by different topological algorithms in the protein-protein interaction network.

\begin{tabular}{|c|c|}
\hline Topological algorithm & Top 20 genes were ranked by score \\
\hline $\begin{array}{l}\text { Maximal Clique } \\
\text { Centrality (MCC) }\end{array}$ & $\begin{array}{c}\text { Btg2, Clu, Csf1, Cx3cl1, Cxcl1, Cxcl5, } \\
\text { Dusp1, Egr1, Egr2, Fos, Ier2, Igf1, Junb, } \\
\text { Kng1, Kng2, Nfkbiz, Penk, Ptgs2, } \\
\text { Serping1, Zfp36 }\end{array}$ \\
\hline Degree & $\begin{array}{c}\text { A2m, Acta2, Clu, Col3a1, Csf1, Cxcl1, } \\
\text { Cxcl5, Dusp1, Egr1, Fos, Igf1, Il7, Kitl, } \\
\text { Kng1, Kng2, Postn, Ptgs2, Socs3, } \\
\text { Vcam1, Vcan }\end{array}$ \\
\hline $\begin{array}{l}\text { Edge Percolated } \\
\text { Component }\end{array}$ & $\begin{array}{l}\text { Cd68, Clu, Cp, Csf1, Cx3cl1, Cxcl1, } \\
\text { Cxcl5, Egr1, Fos, Igf1, Il7, Kitl, Kng1, } \\
\text { Kng2, Nfkbiz, Penk, Ptgs2, Serpina3n, } \\
\text { Socs3, Vcam1 }\end{array}$ \\
\hline $\begin{array}{l}\text { Maximum } \\
\text { Neighborhood } \\
\text { Component }\end{array}$ & $\begin{array}{l}\text { Acta2, Clu, Col3a1, Csf1, Cx3cl1, } \\
\text { Cxcl1, Cxcl5, Dusp1, Egr1, Fos, Igf1, } \\
\text { Il7, Kitl, Kng1, Kng2, Nfkbiz, Postn, } \\
\text { Ptgs2, Serpina3n, Vcam1 }\end{array}$ \\
\hline BottleNeck & $\begin{array}{c}\text { A2m, Acta1, Cd68, Cfh, Cxcl1, Dusp1, } \\
\text { Egr1, Eng, Folr2, Fos, Igf1, Il7, Jag1, } \\
\text { Kitl, Mcam, Negr1, Postn, Ptgs2, } \\
\text { Timp3, Vcam1 }\end{array}$ \\
\hline EcCentricity & $\begin{array}{c}\text { Acta2, Cd68, Csf1, Cx3cl1, Cxcl1, Egr1, } \\
\text { Eng, Fgf10, Fgf9, Fgfr2, Fos, Igf1, Junb, } \\
\text { Lyz2, Mmp12, Myh11, Ptgs2, Serping1, } \\
\text { Smad9, Vcam1 }\end{array}$ \\
\hline $\begin{array}{l}\text { Common genes of } 6 \\
\text { topological algorithms }\end{array}$ & Cxcl1, Egr1, Fos, Igf1, Ptgs2 \\
\hline
\end{tabular}

cellular morphology of notochordal cells (characterized by lots of vacuoles). The culture method in this study was a monolayer with normoxic condition, which may be difficult to preserve the phenotype of notochordal cells due to the phenotype of notochordal cells that would quickly disappear after in vitro culture. Since the optimal culture condition for notochordal cell phenotype maintenance is still under investigation, our study may only represent a part of the mecha-
TAble 8: KEGG and GO analysis of hub gene.

\begin{tabular}{lcc}
\hline Terms & $\begin{array}{c}-\log 10 \\
(P \text { value })\end{array}$ & Count \\
\hline KEGG:rno04668:TNF signaling pathway & 2.937464369 & 3 \\
KEGG:rno05200:Pathways in cancer & 1.8358872 & 3 \\
KEGG:rno04913:Ovarian steroidogenesis & 1.543625084 & 2 \\
KEGG:rno05140:Leishmaniasis & 1.435827789 & 2 \\
KEGG:rno05132:Salmonella infection & 1.375008637 & 2 \\
GO BP:0032496 response to & 4.797953003 & 4 \\
lipopolysaccharide & 3.469546007 & 3 \\
GO BP:0051384 response to glucocorticoid & & \\
GO BP:0042127 regulation of cell & 2.980755828 & 3 \\
proliferation & & \\
GO BP:0014070 response to organic & 2.85105501 & 3 \\
cyclic compound & 2.282716935 & 3 \\
GO BP:0042493 response to drug & & \\
\hline
\end{tabular}

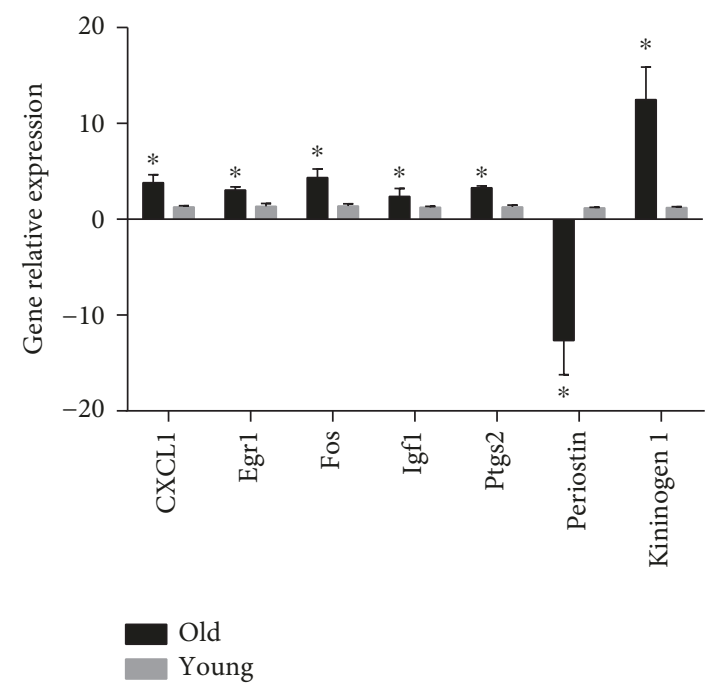

FIgURE 7: Validation of microarray results by RT-PCR. Five hub genes (Cxcl1, Egr1, Fos, Igf1, and Ptgs2) and the most significantly upregulated (kininogen 1) and downregulated (periostin) genes were selected for the real-time PCR validation. GAPDH was selected as the internal control. Data are presented as the mean \pm SD. ${ }^{*} P<0.05$ compared with the young group.

nism of NPC senescence. Another limitation exists in the normoxic culture condition. Hypoxia is one of characteristics of IVD. Previous studies shown that the gene expression of NPCs may be altered due to the normoxic culture condition [55]. Thus, hypoxia and 3 dimensional cultures may be more suitable to maintain the phenotype of NPCs.

\section{Conclusions}

Taken together, we discovered that the old NPCs showed declined proliferation and migration abilities. Furthermore, we identified a series of molecular pathways that contribute to NPC aging and degeneration through microarray analysis. Further studies will be needed to elucidate downstream mechanisms of potential targets. An understanding of the 
mechanisms underlying these aging processes may lead to a novel breakthrough in the prevention and treatment of disc aging and aging-related degeneration.

$\begin{array}{ll}\text { Abbreviations } \\ \text { NP: } & \text { Nucleus pulposus } \\ \text { IVD: } & \text { Intervertebral disc } \\ \text { RT-PCR: } & \text { Real-time polymerase chain reaction } \\ \text { SA- } \beta \text {-gal: } & \text { Senescence-associated } \beta \text {-galactosidase } \\ \text { FITC: } & \text { Fluorescein isothiocyanate } \\ \text { PE: } & \text { Phycoerythrin } \\ \text { EPC: } & \text { Edge Percolated Component } \\ \text { MCC: } & \text { Maximal Clique Centrality } \\ \text { MNC: } & \text { Maximum Neighborhood Component. }\end{array}$

\section{Data Availability}

The data used to support the findings of this study are included within the article and the supplementary materials. The gene microarray datasets had been uploaded to the NCBI Gene Expression Omnibus (GEO) and can be accessed via GEO Series accession [GEO:GSE126883] (https://www .ncbi.nlm.nih.gov/geo/query/acc.cgi?acc=GSE126883). Other related data generated and/or analysed during the current study are available from the corresponding authors upon reasonable request.

\section{Conflicts of Interest}

The authors declare no conflicts of interest.

\section{Authors' Contributions}

Shi Cheng and Xiaochuan Li conceived and designed the experiment. Shi Cheng, Zhiwei Jia, and Yachao Zhao performed experiments, analysed the data, and wrote the manuscript. Linghan Lin provided experimental help and advice. Deli Wang and Dike Ruan supervised the whole process of the research for its accuracy and compliance with institutional regulations. Yu Zhang revised the manuscript. All authors discussed the results and commented on the manuscript. All authors read and approved the final manuscript.

\section{Acknowledgments}

This work was supported by grants from the National Natural Science Foundation of China (81470102 and 81802130) and Research Funding of Peking University Shenzhen Hospital (JCYJ2019003RC).

\section{Supplementary Materials}

Heat map and hierarchical clustering of DEG profile comparison between the young and old NPCs. Red colour indicates high expression, and green colour indicates low expression. Every column represents a tissue sample, and every row represents an mRNA probe. Z641, Z643, and Z642 were samples in the old group; Z644, Z645, and Z646 were samples in the young group. (Supplementary Materials)

\section{References}

[1] J. N. Katz, "Lumbar disc disorders and low-back pain: socioeconomic factors and consequences," The Journal of Bone and Joint Surgery(American), vol. 88, Supplement_2, pp. 2124, 2006.

[2] M. A. Adams and P. J. Roughley, "What is intervertebral disc degeneration, and what causes it?," Spine, vol. 31, no. 18, pp. 2151-2161, 2006.

[3] D. S. Mern, A. Beierfuss, C. Thome, and A. A. Hegewald, "Enhancing human nucleus pulposus cells for biological treatment approaches of degenerative intervertebral disc diseases: a systematic review," Journal of Tissue Engineering and Regenerative Medicine, vol. 8, no. 12, pp. 925-936, 2014.

[4] S. Huang, V. Tam, K. M. C. Cheung et al., "Stem cell-based approaches for intervertebral disc regeneration," Current Stem Cell Research \& Therapy, vol. 6, no. 4, pp. 317-326, 2011.

[5] Y. C. Huang, J. P. G. Urban, and K. D. K. Luk, "Intervertebral disc regeneration: do nutrients lead the way?," Nature reviews Rheumatology, vol. 10, no. 9, pp. 561-566, 2014.

[6] J. Silva-Correia, S. I. Correia, J. M. Oliveira, and R. L. Reis, "Tissue engineering strategies applied in the regeneration of the human intervertebral disk," Biotechnology Advances, vol. 31, no. 8, pp. 1514-1531, 2013.

[7] D. Chen, D. Xia, Z. Pan et al., "Metformin protects against apoptosis and senescence in nucleus pulposus cells and ameliorates disc degeneration in vivo," Cell Death \& Disease, vol. 7, no. 10, article e2441, 2016.

[8] J. J. Trout, J. A. Buckwalter, and K. C. Moore, "Ultrastructure of the human intervertebral disc: II. Cells of the nucleus pulposus," The Anatomical Record, vol. 204, no. 4, pp. 307-314, 1982.

[9] L. Zheng, Y. Cao, S. Ni et al., "Ciliary parathyroid hormone signaling activates transforming growth factor- $\beta$ to maintain intervertebral disc homeostasis during aging," Bone Research, vol. 6, no. 1, article 21, 2018.

[10] F. Wang, F. Cai, R. Shi, X. H. Wang, and X. T. Wu, "Aging and age related stresses: a senescence mechanism of intervertebral disc degeneration," Osteoarthritis and Cartilage, vol. 24, no. 3, pp. 398-408, 2016.

[11] P. Priyadarshani, Y. Li, and L. Yao, "Advances in biological therapy for nucleus pulposus regeneration," Osteoarthritis and Cartilage, vol. 24, no. 2, pp. 206-212, 2016.

[12] D. McHugh and J. Gil, "Senescence and aging: causes, consequences, and therapeutic avenues," The Journal of Cell Biology, vol. 217, no. 1, pp. 65-77, 2018.

[13] J.-S. Lee, "Cellular senescence, aging, and age-related disease: special issue of BMB reports in 2019," BMB Reports, vol. 52, no. 1, pp. 1-2, 2019.

[14] X. Tang, L. Jing, and J. Chen, "Changes in the molecular phenotype of nucleus pulposus cells with intervertebral disc aging," PLoS One, vol. 7, no. 12, article e52020, 2012.

[15] C. Q. Zhao, L. M. Wang, L. S. Jiang, and L. Y. Dai, "The cell biology of intervertebral disc aging and degeneration," Ageing Research Reviews, vol. 6, no. 3, pp. 247-261, 2007.

[16] K. W. Kim, H. N. Chung, K. Y. Ha, J. S. Lee, and Y. Y. Kim, "Senescence mechanisms of nucleus pulposus chondrocytes in human intervertebral discs," The Spine Journal, vol. 9, no. 8, pp. 658-666, 2009.

[17] J. Campisi and F. d'Adda di Fagagna, "Cellular senescence: when bad things happen to good cells," Nature Reviews Molecular Cell Biology, vol. 8, no. 9, pp. 729-740, 2007. 
[18] O. Toussaint, E. E. Medrano, and T. von Zglinicki, "Cellular and molecular mechanisms of stress-induced premature senescence (SIPS) of human diploid fibroblasts and melanocytes," Experimental Gerontology, vol. 35, no. 8, pp. 927-945, 2000.

[19] M. Shawi and C. Autexier, "Telomerase, senescence and ageing," Mechanisms of Ageing and Development, vol. 129, no. 1-2, pp. 3-10, 2008.

[20] A. L. Tarca, R. Romero, and S. Draghici, "Analysis of microarray experiments of gene expression profiling," American Journal of Obstetrics and Gynecology, vol. 195, no. 2, pp. 373-388, 2006.

[21] D. K. Slonim and I. Yanai, "Getting started in gene expression microarray analysis," PLoS Computational Biology, vol. 5, no. 10, article e1000543, 2009.

[22] Y. G. Zhang, Z. M. Sun, J. T. Liu, S. J. Wang, F. L. Ren, and $\mathrm{X}$. Guo, "Features of intervertebral disc degeneration in rat's aging process," Journal of Zhejiang University Science B, vol. 10, no. 7, pp. 522-527, 2009.

[23] M. V. Risbud, Z. R. Schoepflin, F. Mwale et al., "Defining the phenotype of young healthy nucleus pulposus cells: recommendations of the Spine Research Interest Group at the 2014 annual ORS meeting," Journal of Orthopaedic Research, vol. 33, no. 3, pp. 283-293, 2015.

[24] M. Molinos, C. R. Almeida, R. M. Goncalves, and M. A. Barbosa, "Improvement of bovine nucleus pulposus cells isolation leads to identification of three phenotypically distinct cell subpopulations," Tissue Engineering Part A, vol. 21, no. 15-16, pp. 2216-2227, 2015.

[25] N. Fujita, T. Miyamoto, J. Imai et al., "CD24 is expressed specifically in the nucleus pulposus of intervertebral discs," Biochemical and Biophysical Research Communications, vol. 338, no. 4, pp. 1890-1896, 2005.

[26] X. Tang, L. Jing, W. J. Richardson et al., "Identifying molecular phenotype of nucleus pulposus cells in human intervertebral disc with aging and degeneration," Journal of Orthopaedic Research, vol. 34, no. 8, pp. 1316-1326, 2016.

[27] Y. Nakamura, Y. Muguruma, T. Yahata et al., "Expression of CD90 on keratinocyte stem/progenitor cells," The British Journal of Dermatology, vol. 154, no. 6, pp. 1062-1070, 2006.

[28] S. W. Jeong, J. S. Lee, and K. W. Kim, "In vitro lifespan and senescence mechanisms of human nucleus pulposus chondrocytes," The Spine Journal, vol. 14, no. 3, pp. 499-504, 2014.

[29] P. Panchamanon, P. Pavasant, and C. Leethanakul, "Periostin plays role in force-induced stem cell potential by periodontal ligament stem cells," Cell Biology International, vol. 43, no. 5, pp. 506-515, 2019.

[30] Z. Wu, W. Dai, P. Wang et al., "Periostin promotes migration, proliferation, and differentiation of human periodontal ligament mesenchymal stem cells," Connective Tissue Research, vol. 59, no. 2, pp. 108-119, 2018.

[31] O. Duchamp de Lageneste, A. Julien, R. Abou-Khalil et al., "Periosteum contains skeletal stem cells with high bone regenerative potential controlled by periostin," Nature Communications, vol. 9, no. 1, article 773, 2018.

[32] A. Graja, F. Garcia-Carrizo, A.-M. Jank et al., "Loss of periostin occurs in aging adipose tissue of mice and its genetic ablation impairs adipose tissue lipid metabolism," Aging Cell, vol. 17, no. 5, article e12810, 2018.

[33] T. Chow and I. M. Rogers, "Periostin is critical for improving the therapeutic properties of adipocyte-derived stem cells," Stem Cell Research \& Therapy, vol. 6, no. 1, article 214, 2015.
[34] A. Kudo, "Periostin in fibrillogenesis for tissue regeneration: periostin actions inside and outside the cell," Cellular and Molecular Life Sciences, vol. 68, no. 19, pp. 3201-3207, 2011.

[35] M. Egbert, M. Ruetze, M. Sattler et al., "The matricellular protein periostin contributes to proper collagen function and is downregulated during skin aging," Journal of Dermatological Science, vol. 73, no. 1, pp. 40-48, 2014.

[36] H. E. Gruber, R. A. Norris, M. J. Kern et al., "Periostin is expressed by cells of the human and sand rat intervertebral discs," Biotechnic \& Histochemistry, vol. 86, no. 3, pp. 199206, 2011.

[37] T. T. Tsai, P. L. Lai, J. C. Liao et al., "Increased periostin gene expression in degenerative intervertebral disc cells," The Spine Journal, vol. 13, no. 3, pp. 289-298, 2013.

[38] I. M. Sainz, I. Isordia-Salas, J. L. Castaneda et al., "Modulation of inflammation by kininogen deficiency in a rat model of inflammatory arthritis," Arthritis and Rheumatism, vol. 52, no. 8, pp. 2549-2552, 2005.

[39] I. Isordia-Salas, R. A. Pixley, I. M. Sainz, C. Martinez-Murillo, and R. W. Colman, "The role of plasma high molecular weight kininogen in experimental intestinal and systemic inflammation," Archives of Medical Research, vol. 36, no. 1, pp. 87-95, 2005.

[40] C. H. Lin, C. C. Liao, C. H. Huang et al., "Proteomics analysis to identify and characterize the biomarkers and physical activities of non-frail and frail older adults," International Journal of Medical Sciences, vol. 14, no. 3, pp. 231-239, 2017.

[41] Y. W. Lam, N. N. C. Tam, J. E. Evans, K. M. Green, X. Zhang, and S. M. Ho, "Differential proteomics in the aging Noble rat ventral prostate," Proteomics, vol. 8, no. 13, pp. 2750-2763, 2008.

[42] J. Dai, X. Zhu, M. C. Yoder, Y. Wu, and R. W. Colman, "Cleaved high-molecular-weight kininogen accelerates the onset of endothelial progenitor cell senescence by induction of reactive oxygen species," Arteriosclerosis, Thrombosis, and Vascular Biology, vol. 31, no. 4, pp. 883-889, 2011.

[43] K. Li, Y. Li, B. Xu, L. Mao, and J. Zhao, "Sesamin inhibits lipopolysaccharide-induced inflammation and extracellular matrix catabolism in rat intervertebral disc," Connective Tissue Research, vol. 57, no. 5, pp. 347-359, 2016.

[44] T. Kadow, G. Sowa, N. Vo, and J. D. Kang, "Molecular basis of intervertebral disc degeneration and herniations: what are the important translational questions?," Clinical Orthopaedics and Related Research, vol. 473, no. 6, pp. 19031912, 2015.

[45] S. Wang, J. Wei, Y. Fan et al., "Progranulin is positively associated with intervertebral disc degeneration by interaction with IL-10 and IL-17 through TNF pathways," Inflammation, vol. 41, no. 5, pp. 1852-1863, 2018.

[46] Z. I. Johnson, Z. R. Schoepflin, H. Choi, I. M. Shapiro, and M. V. Risbud, "Disc in flames: roles of TNF- $\alpha$ and IL- $1 \beta$ in intervertebral disc degeneration," European Cells and Materials, vol. 30, pp. 104-117, 2015.

[47] Z. Tian, X. Ma, M. Yasen et al., "Intervertebral disc degeneration in a percutaneous mouse tail injury model," American Journal of Physical Medicine \& Rehabilitation, vol. 97, no. 3, pp. 170-177, 2018.

[48] C. A. Seguin, R. M. Pilliar, J. A. Madri, and R. A. Kandel, "TNF-alpha induces MMP2 gelatinase activity and MT1MMP expression in an in vitro model of nucleus pulposus tissue degeneration," Spine, vol. 33, no. 4, pp. 356-365, 2008. 
[49] T. Yoshida, J. S. Park, K. Yokosuka et al., "Effect of a nonprotein bioactive agent on the reduction of cyclooxygenase2 and tumor necrosis factor- $\alpha$ in human intervertebral disc cells in vitro," Journal of Neurosurgery: Spine, vol. 9, no. 5, pp. 411-418, 2008.

[50] J. Tolonen, M. Grönblad, J. Virri, S. Seitsalo, T. Rytömaa, and E. Karaharju, "Oncoprotein c-Fos and c-Jun immunopositive cells and cell clusters in herniated intervertebral disc tissue," European Spine Journal, vol. 11, no. 5, pp. 452-458, 2002.

[51] Y. He, W. Zhu, M. H. Shin et al., "cFOS-SOX9 axis reprograms bone marrow-derived mesenchymal stem cells into chondroblastic osteosarcoma," Stem Cell Reports, vol. 8, no. 6, pp. 1630-1644, 2017.

[52] A. Tsarouhas, G. Soufla, K. Tsarouhas et al., "Molecular profile of major growth factors in lumbar intervertebral disc herniation: correlation with patient clinical and epidemiological characteristics," Molecular Medicine Reports, vol. 15, no. 4, pp. 2195-2203, 2017.

[53] R. F. Loeser, G. Shanker, C. S. Carlson, J. F. Gardin, B. J. Shelton, and W. E. Sonntag, "Reduction in the chondrocyte response to insulin-like growth factor 1 in aging and osteoarthritis: studies in a non-human primate model of naturally occurring disease," Arthritis and Rheumatism, vol. 43, no. 9, pp. 2110-2120, 2000.

[54] S. Okuda, A. Myoui, K. Ariga, T. Nakase, K. Yonenobu, and $\mathrm{H}$. Yoshikawa, "Mechanisms of age-related decline in insulin-like growth factor-I dependent proteoglycan synthesis in rat intervertebral disc cells," Spine, vol. 26, no. 22, pp. 24212426, 2001.

[55] S. H. Yang, M. H. Hu, W. Y. Lo, Y. H. Sun, C. C. Wu, and K. C. Yang, "The influence of oxygen concentration on the extracellular matrix production of human nucleus pulposus cells during isolation-expansion process," Journal of Biomedical Materials Research Part A, vol. 105, no. 6, pp. 1575-1582, 2017. 


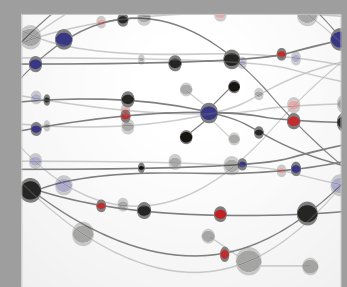

The Scientific World Journal
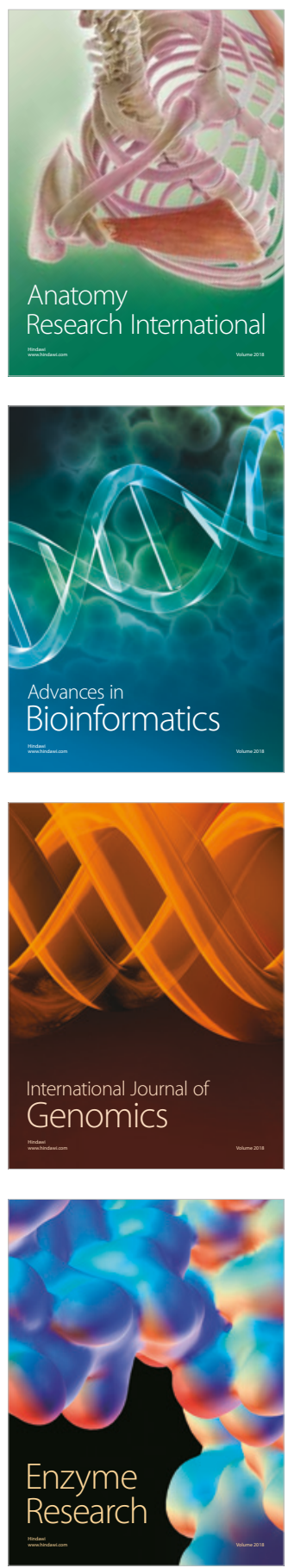
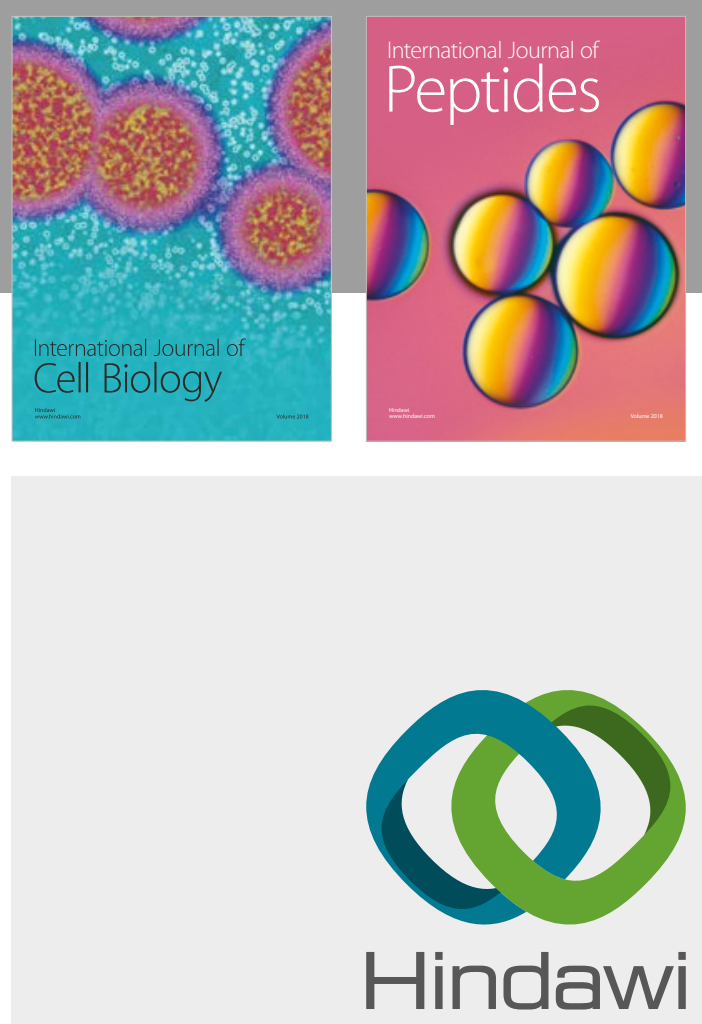

Submit your manuscripts at

www.hindawi.com
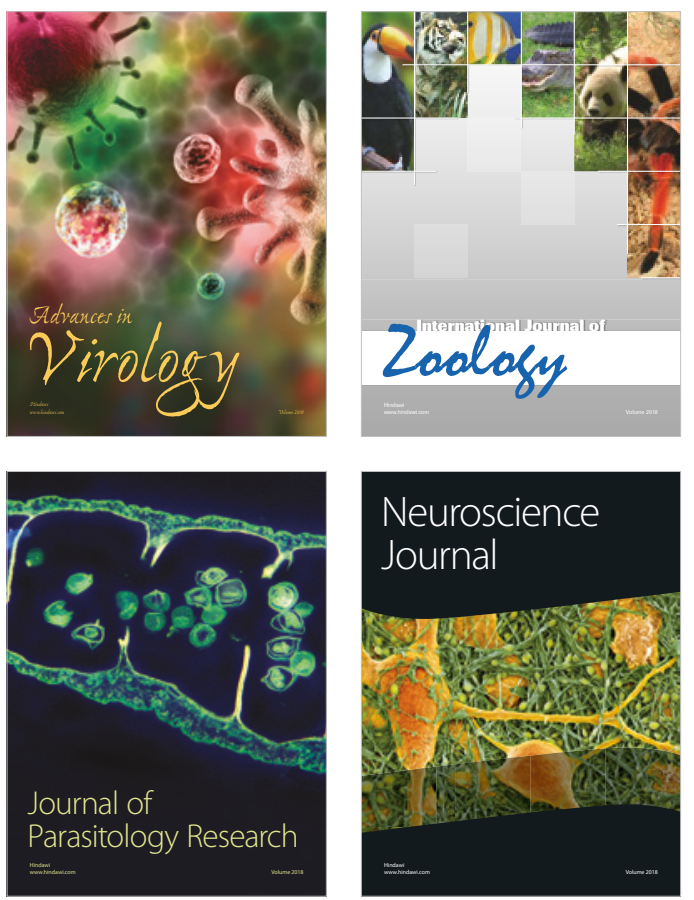
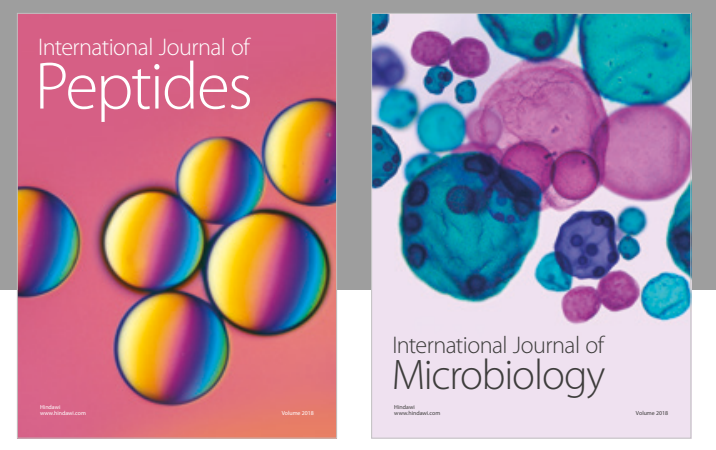

nternational Journal of Microbiology
Journal of
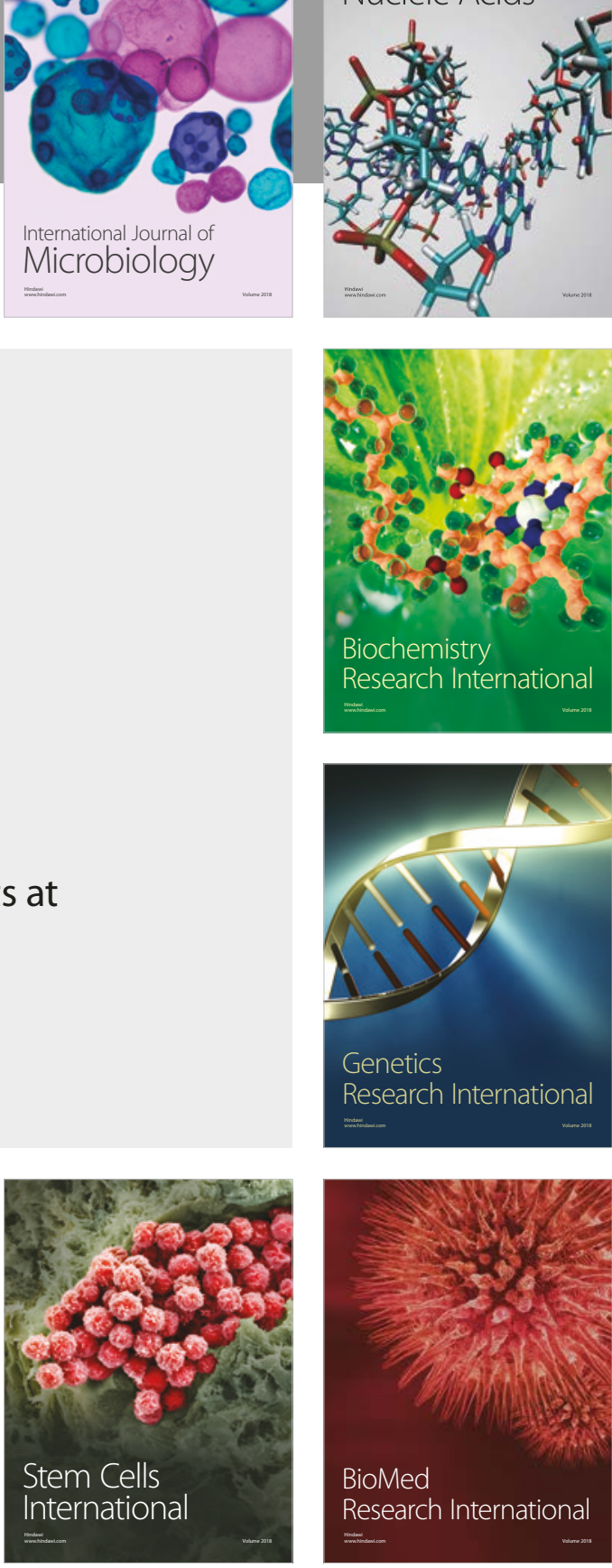
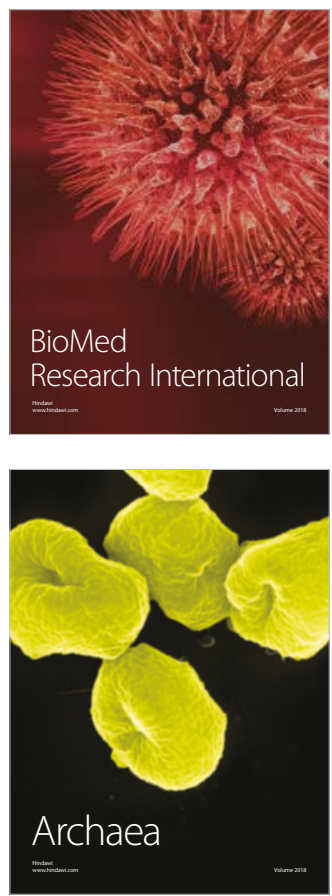